University of South Florida

DIGITAL COMMONS

Digital Commons @ University of

@ UNIVERSITY OF SOUTH FLORIDA

South Florida

Marine Science Faculty Publications

College of Marine Science

$1-1985$

\title{
Mixed Instabilities in the Gulf Stream over the Continental Slope
}

Mark E. Luther

University of North Carolina, mluther@usf.edu

John M. Bane

University of North Carolina

Follow this and additional works at: https://digitalcommons.usf.edu/msc_facpub

Part of the Life Sciences Commons

\section{Scholar Commons Citation}

Luther, Mark E. and Bane, John M., "Mixed Instabilities in the Gulf Stream over the Continental Slope" (1985). Marine Science Faculty Publications. 506.

https://digitalcommons.usf.edu/msc_facpub/506

This Article is brought to you for free and open access by the College of Marine Science at Digital Commons @ University of South Florida. It has been accepted for inclusion in Marine Science Faculty Publications by an authorized administrator of Digital Commons @ University of South Florida. For more information, please contact digitalcommons@usf.edu. 


\title{
Mixed Instabilities in the Gulf Stream over the Continental Slope
}

\author{
MARK E. LuTHER $†$ AND JOHN M. BANE, JR. \\ Marine Sciences Program, The University of North Carolina, Chapel Hill, NC 27514
}

(Manuscript received 28 December 1982, in final form 10 September 1984)

\begin{abstract}
A numerical model study is presented of the unstable normal modes of oscillation of a boundary current. The model background current approximates the Gulf Stream south of Cape Hatteras, North Carolina. Both vertical and horizontal shear in current velocity and a sloping bottom topography are included. The study seeks small amplitude, alongshore propagating perturbations with real frequency and complex alongshore wavenumber. A nonzero imaginary part of the wavenumber ensures that the wave amplitude either grows or decays in the alongshore direction. The first four eigenmodes are identified and their dispersion relations are investigated. Higher order modes are not resolved by the model. The dispersion surfaces (eigenvalues of frequency as a function of complex wavenumber) appear to bifurcate with increasing values of real wave number.

Observations in the Gulf Stream south of Cape Hatteras have revealed a persistent wave-like meander pattern in the Stream with a period of 7-8 days. This wave form propagates in the downstream direction with a phase speed of about $40 \mathrm{~km} \mathrm{day}^{-1}$ and is uncorrelated with local wind forcing. An 8-day wave also appears as an eigenmode in the model, and the perturbation velocity and buoyancy fields are consistent with observations. The instability mechanism of the model wave is of the mixed barotropic-baroclinic type, with the majority (about $80 \%$ ) of the perturbation energy derived from the potential energy of the background flow. The model 8-day wave consists of a side-to-side meandering of the core of the current with filamentlike structures of warm water (positive perturbation buoyancy) trailing the shoreward-most excursion of the core of the current. These filaments are separated from the core of the current by a cold dome of upwelled water.
\end{abstract}

\section{Introduction}

Wavelike meanders in the path of the Gulf Stream have been observed to be the dominant mode of oscillation in the Stream from the Florida Straits to Cape Hatteras. These meanders propagate to the northeast along the Stream with periods on the order of one week, alongshore wavelengths of a few hundred kilometers, and cross-Stream amplitudes of a few tens of kilometers. They play an important role in the mass and energy exchanges between deep ocean and continental shelf waters.

Webster (1961a) described the meander process off Onslow Bay, North Carolina, as a skewed wavelike motion with a period of approximately seven days. He noted that the meanders were not related to any apparent forcing. Energetics calculations for the meanders (Webster, 1961b) suggested that the energy transfer was from the meanders to the mean flow in the area off Onslow Bay.

Legeckis (1979) studied the meanders from the Florida Straits to Cape Hatteras using satellite infrared

$\dagger$ Present affiliation: Mesoscale Air-Sea Interaction Group, Meteorology Annex, The Florida State University, Tallahassee, FL 32306. imagery of the sea surface. He found that the meanders propagated along the Stream to the northeast (the downstream direction) at an average phase speed of $40 \mathrm{~km} \mathrm{day}{ }^{-1}$ with an average wavelength of about $150 \mathrm{~km}$. Legeckis (1979) and Bane and Brooks (1979) independently found that the amplitude of the meanders increases dramatically downstream of a bottom topographic feature off Charleston, South Carolina, known as the "Charleston Bump." Several authors have suggested that the seaward deflection of the Gulf Stream off Charleston is caused by the Bump (Pashinski and Maul, 1973; Legeckis, 1976, 1979; Brooks and Bane, 1978) and that this deflection is responsible for the initiation of the increased meander amplitude downstream of the Charleston Bump (Legeckis, 1976, 1979; Bane and Brooks, 1979; Chao and Janowitz, 1979; Brooks and Bane, 1981; Bane et al., 1981).

The meanders downstream of Charleston were described extensively by Brooks and Bane (1981) and by Bane et al. (1981) on the basis of data from a simultaneous moored instrument array and an airdeployed expendable bathythermograph (AXBT) survey. Energy spectra from the current meter records showed prominent peaks at the 7-8 day period. Phase lags in this period band give wavelengths of approximately $230 \mathrm{~km}$, with downstream phase speeds of 
32-35 km day ${ }^{-1}$. The nearsurface temperature structures of two meanders were mapped extensively by the AXBT survey as they propagated through the study area from Charleston to Cape Hatteras (Fig. 1). Both meanders exhibited warm filament-like structures trailing behind a meander crest (the shoreward-most excursion of the Stream). The warm filaments were separated from the main body of the Stream by a dome of cooler water upwelled from deep within or below the Stream. These features are quite similar to

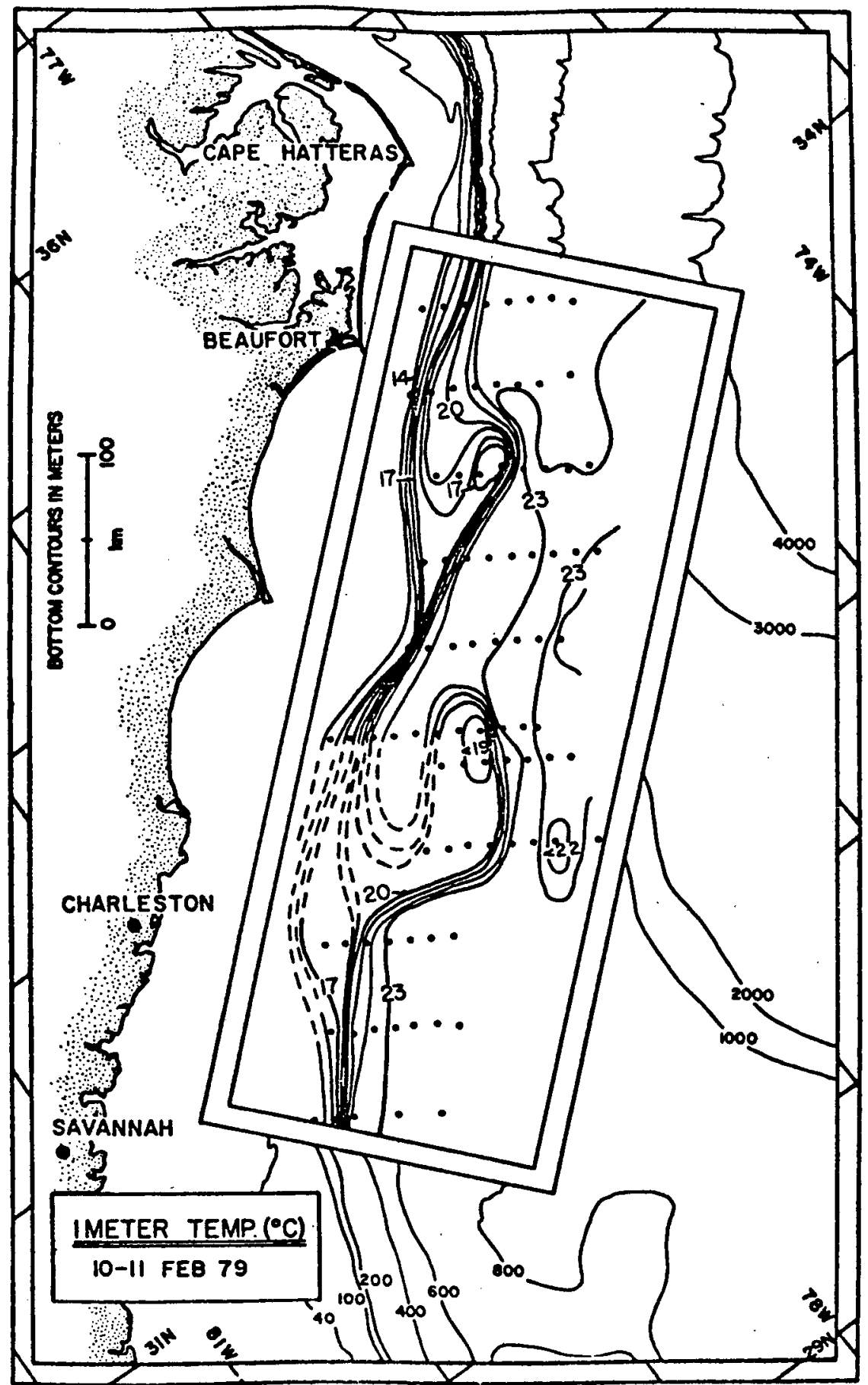

FIG. 1. Inner box shows contours of temperature at $1 \mathrm{~m}$ depth off the Carolina Coast, as determined from an AXBT survey on 10-11 February 1979. Outer box shows bottom topography (from Bane et al., 1981). Note the strong seaward deflection of the thermal front off Charleston, and the two meanders with trailing filaments downstream. 
those observed by Lee et al. (1981) in the area upstream of the Charleston Bump, but are of larger amplitude. Brooks and Bane (1981) found no correlation between the velocity fluctuations in the 7-8 day period band and local wind stress or coastal sea level fluctuations.

The repeated appearance of this 7-8 day period meander (henceforth called the 8-day wave) in the absence of any consistent forcing suggests that it is a natural mode of oscillation of the Gulf Stream. The rapid growth of these waves downstream of the Charleston Bump indicates that they may be the result of an inherent instability in the Stream triggered by the Bump. Since the Gulf Stream possesses both horizontal and vertical current shear, there is the possibility of both barotropic instability (conversion of mean kinetic energy to fluctuation energy) and baroclinic instability (conversion of mean potential energy to fluctuation energy).

The theory of quasi-geostrophic baroclinic instability is by now well understood (Charney, 1947; Eady, 1949). Pedlosky (1964a) derived the necessary conditions for instability from potential vorticity considerations. The role of critical levels and the vorticity dynamics of baroclinic instability were investigated by Bretherton (1966a,b). Numerous authors have investigated the general mixed baroclinic-barotropic instability problem (Pedlosky, 1964b; McIntyre, 1970; Hart, 1974; Gent, 1975; Killworth, 1980; Holland and Haidvogel, 1980; Wright, 1980). Others have considered the stability of currents over bottom topography (Orlanski, 1969; Orlanski and Cox, 1973; de Szoeke, 1975; Mysak et al., 1981). Most of these studies have employed a quasi-geostrophic approximation and have used very simple background fields in their stability analysis. Orlanski and Cox (1973), in a time-dependent primitive equation model, did not make a quasigeostrophic approximation but did include realistic background fields as an initial condition; however, they did not include the effects of a wide continental shelf, as is found off the Carolinas, nor did they use realistic values for the horizontal shear and mass transport of the Gulf Stream in this region. They did include nonlinear effects and found that the growth rate for the disturbances was decreased by an order of magnitude over that predicted by linear theory. They found that the phase speed for the unstable waves was equal to the average of the background current speed. The initial value approach used by Orlanski and Cox (1973) leads to complicated results that are somewhat difficult to interpret, and is not consistent with the idea of an instability triggered by topography.

Hogg (1976) showed that spatially growing waves with complex wavenumber and real frequency can exist in a baroclinic flow and that these waves may be forced by bottom topography. The spatially growing waves were found to be quite different from the usual temporally growing waves. In the present study, the concept of spatially growing waves is used to model the unstable normal modes of oscillation of the Gulf Stream. We use background fields that approximate as closely as possible the Gulf Stream off the Carolina coast, and bottom topography approximating that off Onslow Bay, North Carolina. Because of the large variations in topography and the presence of a strong density front in the background state, we do not make any assumption of quasigeostrophy (cf. Pedlosky, 1979, Section 8.1). Spatially unstable normal modes are found, illustrating at least the incipient stages of meander development that might be triggered by a topographic feature such as the Charleston Bump.

\section{Formulation}

We consider wave motions superimposed on a steady alongshore flow over a sloping bottom topography. The background velocity field is in geostrophic and hydrostatic balance with the mean density field. A straight coastline is assumed along the $y$-axis, with the $x$-axis pointing in the offshore direction and the $z$-axis pointing vertically upward (Fig. 2 ). The depth $h(x)$ is monotonically increasing in the seaward direction. All background quantities are uniform in $y$ and continuous in $x$ and $z$. The fluid is assumed to be inviscid, incompressible, nondiffusive, and continuously density-stratified. Variations in the Coriolis parameter $f$ are neglected.

The equations of motion for a hydrostatic, Boussinesq fluid are linearized about the background state. The equations governing the wave motion are:

\section{MODEL GEOMETRY}

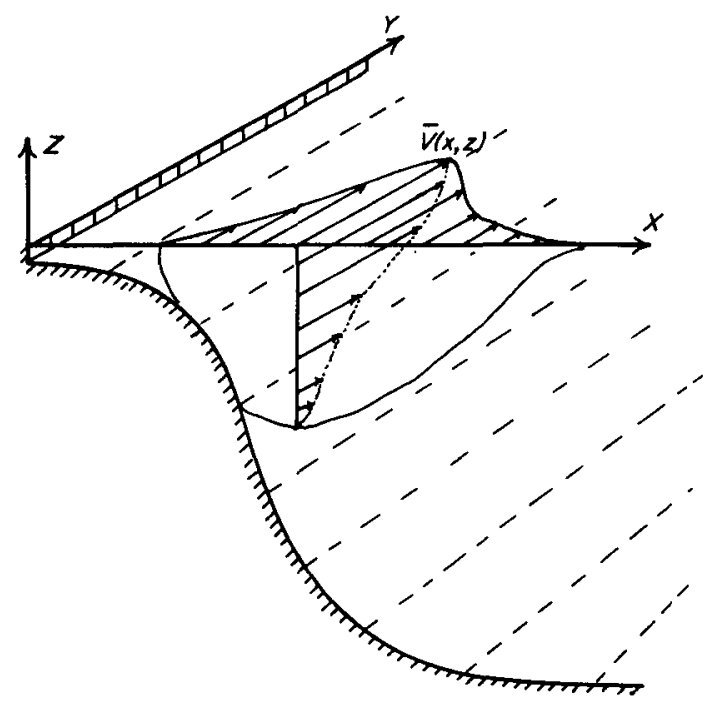

FIG. 2. Schematic of model geometry. 


$$
\begin{gathered}
\frac{D \tilde{u}}{D t}-f \tilde{v}=-\tilde{\pi}_{x}, \\
\frac{D \tilde{v}}{D t}+\left(f+V_{x}\right) \tilde{u}+V_{z} \tilde{w}=-\tilde{\pi}_{y}, \\
0=-\tilde{\pi}_{z}+\tilde{b}, \\
\tilde{u}_{x}+\tilde{v}_{y}+\tilde{w}_{z}=0, \\
\frac{D \tilde{b}}{D t}+M^{2} \tilde{u}+N^{2} \tilde{w}=0,
\end{gathered}
$$

where

$$
\frac{D}{D t}=\frac{\partial}{\partial t}+V(x, z) \frac{\partial}{\partial y},
$$

$(\tilde{u}, \tilde{v}, \tilde{w})$ are the $(x, y, z)$ components of the perturbation velocity, $\tilde{\pi}$ is the pertubation pressure per unit density, $\tilde{b}$ the pertubation buoyancy, $V$ the $y$ component of background velocity (the other components vanish), $N^{2}$ is the Brunt-Väisälä or buoyancy frequency and $M^{2}$ is its horizontal analog (Mooers, 1975). If $B=-\mathrm{g} \bar{\rho}(x, z) / \rho_{0}$ is the background buoyancy, where $g$ is the acceleration of gravity, $\bar{\rho}$ the background density and $\rho_{0}$ a reference density, then:

$$
\begin{aligned}
& N^{2}=B_{z}, \\
& M^{2}=B_{x} .
\end{aligned}
$$

The background flow is governed by

$$
\begin{gathered}
f V=\Pi_{x}, \\
\Pi_{z}=B,
\end{gathered}
$$

where $\Pi$ is the background pressure per unit density. Together, (2.3a) and (2.3b) yield the thermal wind relation

$$
f V_{z}=M^{2} \text {. }
$$

The strong horizontal density gradients encountered in realistic background fields require the inclusion of $M^{2}$ in (2.1e), and $V_{z}$ is also included in (2.1b) because of the strong vertical shears implied by (2.4). The presence of density fronts in the background state and the inclusion of a continental shelf topography preclude the use of a quasigeostrophic approximation.

Since the coefficients of (2.1) are independent of alongshore (along-stream) distance $y$ and time $t$, solutions are sought in the form of alongshore propagating waves; therefore, the perturbation variables are assumed to be of the form

$$
(\tilde{u}, \tilde{v}, \tilde{w}, \tilde{\pi}, \tilde{b})=\operatorname{Re}\left[(u, v, w, \pi, b) e^{i(\sigma o t+l y)}\right]
$$

where $\operatorname{Re}[\quad]$ denotes the real part of the expression in brackets, $i=(-1)^{1 / 2}, \sigma_{0}$ is the wave frequency and $l$ the alongshore wave number. The amplitudes $(u, v$, $w, \pi, b)$ are thus complex functions of $x$ and $z$. The intrinsic (or Doppler shifted) frequency, i.e., the frequency relative to the background flow, is given by

$$
\sigma(x, z)=\sigma_{0}+V(x, z) l .
$$

$D / D t$ and $\partial / \partial y$ and (2.1) are thus replaced by $i \sigma$ and il respectively.

All quantities are scaled by their reference values as follows:

$$
\begin{gathered}
\left(x^{\prime}, y^{\prime}\right)=(x, y) / L, \quad z^{\prime}=z / H, \quad h^{\prime}=h / H, \\
\left(u^{\prime}, v^{\prime}\right)=(u, v) u_{0}, \quad w^{\prime}=\frac{L}{H} w / u_{0}, \\
\pi^{\prime}=\pi / u_{0} f L, \quad b^{\prime}=\frac{H}{u_{0} f L} b, \\
\sigma^{\prime}=\frac{\sigma}{f}=\sigma_{0}^{\prime}+R_{0} V^{\prime} l^{\prime}, \quad l^{\prime}=l L, \quad \sigma_{0}^{\prime}=\sigma_{0} / f,
\end{gathered}
$$

$N^{2 \prime}=N^{2} / N_{0}^{2}, \quad M^{2 \prime}=M^{2} / M_{0}^{2}, \quad V^{\prime}=V / V_{0}$,

where $L$ is a horizontal length scale, $H$ the maximum depth, $V_{0}, N_{0}^{2}$ and $M_{0}^{2}$ are the maximum values of $V, N^{2}$ and $M^{2}, u_{0}$ is a typical perturbation velocity scale (determined by the forcing), and $R_{0}=V_{0} /(f L)$ is the background Rossby number.

We follow the customary practice of substituting the dimensionless variables and immediately dropping the primes. We then eliminate the buoyancy $b$ between (2.1e) and (2.1c) and solve for $u, v$ and $w$ in terms of $\pi$ to give

$$
\begin{gathered}
u=\frac{i}{\Delta}\left[-\sigma N^{2} \pi_{x}+\alpha \sigma M^{2} \pi_{z}-l N^{2} \pi\right], \\
v=\frac{1}{\Delta}\left[\left(N^{2} \sigma_{f}^{2}-\alpha^{2} S^{2} M^{4}\right) \pi_{x}-\alpha \sigma^{2} M^{2} \pi_{z}+\sigma l N^{2} \pi\right], \\
w=\frac{i}{\Delta}\left[\alpha \sigma M^{2} \pi_{x}-\frac{\sigma\left(\sigma_{f}^{2}-\sigma^{2}\right)}{S^{2}} \pi_{z}+\alpha l M^{2} \pi\right],
\end{gathered}
$$

where $\Delta=N^{2}\left(\sigma_{f}^{2}-\sigma^{2}\right)-\alpha^{2} S^{2} M^{4}, \sigma_{f}^{2}=1+R_{0} V_{x}$, $S^{2}=N_{0}^{2} H^{2} /\left(f^{2} L^{2}\right)$ is a stratification parameter or Burger number and is the square of the ratio of the internal Rossby radius $R_{i}=N_{0} H / f$ to the horizontal length scale $L$, and $\alpha=M_{0} L /\left(N_{0}^{2} H\right)$ is an "aspect ratio" for the background density field.

Equations (2.7) are substituted into (2.1d) to give a governing equation for $\pi$. A coordinate transformation $\zeta=z / h(x)$ is made to facilitate the application of boundary conditions. Then the transformed governing equation is

$$
C_{x x} \pi_{x x}+C_{x \zeta} \pi_{x \zeta}+C_{\zeta \zeta} \pi_{\zeta \zeta}+C_{x} \pi_{x}+C_{\zeta} \pi_{\zeta}+C_{F} \pi=0,
$$

where

$$
\begin{gathered}
C_{x x}=1, \\
C_{x \zeta}=\frac{-2}{h}\left(h_{x} \zeta+\alpha \frac{M^{2}}{N^{2}}\right),
\end{gathered}
$$




$$
\begin{gathered}
C_{\zeta \zeta}=\frac{1}{h^{2}}\left[h_{x}^{2} \zeta^{2}+2 \alpha \frac{M^{2}}{N^{2}} h_{x} \zeta+\frac{\left(\sigma_{f}^{2}-\sigma^{2}\right)}{S^{2} N^{2}}\right], \\
C_{x}=\frac{1}{\Delta}\left(\alpha \frac{M^{2}}{N^{2}} \frac{\partial \Delta}{\partial z}-\frac{\partial \Delta}{\partial x}\right), \\
C_{\zeta}=\frac{1}{h}\left\{\left[2 \frac{h_{x}^{2}}{h}-h_{x x}+\frac{h_{x}}{\Delta}\left(\frac{\partial \Delta}{\partial x}-\alpha \frac{M^{2}}{N^{2}} \frac{\partial \Delta}{\partial z}\right)\right] \zeta\right. \\
+\frac{1}{\Delta}\left(\alpha \frac{M^{2}}{N^{2}} \frac{\partial \Delta}{\partial x}-\frac{\left(\sigma_{f}^{2}-\sigma^{2}\right)}{S^{2} N^{2}} \frac{\partial \Delta}{\partial z}\right) \\
\left.+2 \alpha \frac{M^{2}}{N^{2}}\left(\frac{h_{x}}{h}-\sigma l\right)\right\}, \\
C_{F}=\frac{l}{\sigma \Delta}\left(\alpha \frac{M^{2}}{N^{2}} \frac{\partial \Delta}{\partial x}-\frac{\partial \Delta}{\partial z}\right)-l^{2} .
\end{gathered}
$$

The boundary conditions appropriate for such wave motions are as follows. At the coast, $x=0$, a vertical wall is imposed $[h(0) \neq 0]$ so that there is no flow normal to the shoreline; or that $u=0$. From (2.7a) this gives

$$
\pi_{x}-\left(\alpha \frac{M^{2}}{N^{2}}+\frac{\zeta h_{x}}{h}\right) \pi_{\zeta}+\frac{l \pi}{\sigma}=0 \quad \text { at } \quad x=0 .
$$

There is no flow normal to the bottom, so that $w$ $=-u h_{x}$ at $\zeta=-1$. From $(2.7 \mathrm{a}, \mathrm{c})$ this gives

$$
\begin{aligned}
&\left(\alpha \frac{M^{2}}{N^{2}}-h_{x}\right) \pi_{x}+\frac{1}{h}\left[2 \alpha \frac{M^{2}}{N^{2}} h_{x}-h_{x}^{2}\right. \\
&\left.-\left(\frac{\sigma_{f}^{2}-\sigma^{2}}{S^{2} N^{2}}\right)\right] \pi_{\zeta}+\frac{l}{\sigma}\left(\alpha \frac{M^{2}}{N^{2}}-h_{x}\right) \pi=0 \\
& \text { at } \zeta=-1 .
\end{aligned}
$$

Far offshore, the perturbation pressure vanishes:

$$
\pi \rightarrow 0 \text { as } x \rightarrow \infty \text {. }
$$

The system is forced at the top boundary by an alongshore propagating wind stress curl with the same frequency and complex wave number as the solution, which results in a vertical velocity at the top due to Ekman pumping: $w=\tau$ at $\zeta=0$. For the homogeneous (unforced) problem, $\tau=0$, and this boundary condition becomes the rigid lid approximation. From $(2.7 \mathrm{c})$,

$$
\begin{gathered}
\alpha M^{2} \pi_{x}-\frac{\sigma_{f}^{2}-\sigma^{2}}{S^{2} h} \pi_{\zeta}+\alpha \frac{l}{\sigma} M^{2} \pi=\frac{-i \Delta}{\sigma} \tau \\
\text { at } \zeta=0 .
\end{gathered}
$$

Equations (2.8) and (2.9) form a normal mode problem with $\pi$ as an eigenfunction and $\left(\sigma_{0}, l\right)$ as eigenvalues of the system. Generally, $\sigma_{0}$ is a complex function of the complex wave number $l$ for each mode. In the usual stability analysis, however, $l$ is required to be real, and complex eigenvalues for $\sigma_{0}$ are sought for which $\operatorname{Im}\left\{\sigma_{0}\right\}<0$. This results in modes which grow exponentially in time, illustrating an instability of the basic flow which might be excited by an initial disturbance with real wave number. In the Gulf Stream off the southeast coast of the United States, the waves appear to be excited at a fixed location in space, the Charleston Bump, and to grow as they propagate away from the Bump. This situation is analogous to laboratory demonstrations of instability in which a fixed wave maker vibrating with real frequency generates disturbances that grow as they propagate away from their source. In such cases it is appropriate to consider disturbances with complex wavenumber and real frequency (Watson, 1962; Gaster, 1962, 1965). It has been shown that spatially growing waves can exist in a baroclinic shear flow and are substantially different from temporally growing waves (Hogg, 1976).

For a fixed value of the complex wave number $l$, nontrivial solutions to the homogeneous problem will exist only for certain values of the real frequency $\sigma_{0}$. This follows from the linearity of (2.8) and the boundary conditions (2.9). The forced problem, however, will have a nontrivial solution for any value of $\sigma_{0}$, but the forced response will be small unless the forcing frequency is near an eigenfrequency. The eigenvalues are located by varying the forcing frequency, while keeping all other parameters fixed, and by searching for local maxima of the response in frequency space. The measure of response chosen is the integrated total energy of the disturbance in the $(x, z)$ plane at $y=0$ :

$$
E=\frac{1}{2} \int_{0}^{\infty} \int_{-h}^{0}|u|^{2}+|v|^{2}+\frac{H^{2}}{L^{2}}|w|^{2}+\frac{|b|^{2}}{S^{2} N^{2}} d z d x
$$

The forced response to (2.8) and (2.9) may contain other modes in which the normal modes are embedded. This problem has been noted in similar studies (Schoeberl and Clark, 1980; Salby, 1981). It can easily be shown that the forced response is overwhelmed by the normal mode, as long as the forcing frequency is near the frequency of the normal mode and the spatial structure of the forcing has some projection into the normal mode structure (Salby, 1981). The problem of locating the normal modes for fixed complex wavenumber thus reduces to that of finding the eigenvalues of the single parameter $\sigma_{0}$. The exact spatial structure of the forcing is relatively unimportant.

The normal modes are of the form

$$
\tilde{\pi}=\operatorname{Re}\left[\pi(x, \zeta) e^{i\left(\sigma_{0} t+l y\right)}\right]
$$

where $\operatorname{Re}[$ ] denotes the real part of the expression. Since both $\pi$ and $l$ are complex, this expression can be rewritten as

$$
\tilde{\pi}=e^{-l i y}|\pi(x, \zeta)| \cos \left[\sigma_{0} t+l_{r} y+\theta(x, \zeta)\right],
$$


where subscripts $r$ and $i$ denote the real and imaginary parts of a complex number, and

$$
\left.\begin{array}{l}
|\pi|=\left(\pi_{r}^{2}+\pi_{i}^{2}\right)^{1 / 2} \\
\theta=\tan ^{-1}\left(\pi_{i} / \pi_{r}\right)
\end{array}\right\}
$$

Thus, if normal modes can be found corresponding to negative values of $l_{i}$, then those modes will grow exponentially in the alongshore direction. Of course, after the disturbance has traveled a sufficient distance in the positive $y$-direction, it will become large enough that nonlinear effects will become important, so that (2.8) and (2.9) will no longer accurately describe the motion. Still, the existence of normal modes that grow in the alongshore direction would illustrate an instability of the basic state that might be triggered by a stationary forcing mechanism, such as topography. The growth rates of the different normal modes will indicate which modes are favored by the instability mechanism.

The complicated form of (2.8) and (2.9) makes it difficult, if not impossible, to find simple integral constraints on the phase speed and growth rates for the unstable modes. Quasi-geostrophic theory has shown (cf. Pedlosky, 1979, Section 7.5) that in the absence of a planetary vorticity gradient, the real part of the wave phase speed must lie in the range of the background current speed for the wave to be unstable:

$$
V_{\min } \leqslant \frac{-\sigma_{0}}{l_{r}} \leqslant V_{\max } .
$$

Unstable waves must therefore have a critical level. Conversely, it can be shown that no stable waves can exist that have a critical level unless the background potential vorticity gradient vanishes at the wave's critical level (Bretherton, 1966a). This is only an approximate bound on the phase speed, since it was derived from quasi-geostrophic theory for temporally growing waves and does not include the effects of bottom topography or variable density along a horizontal boundary. The presence of density gradients along rigid horizontal boundaries can be thought of as "gradients of potential vorticity concentrated in sheets just within the fluid" (Bretherton, 1966a). Thus, the presence of density gradients along the surface as well as the sloping bottom topography provide mechanisms for vortex stretching in addition to the variations in isopycnal slope in the interior.

Inspection of the coefficients of (2.8) and (2.9) reveals that $\pi\left(x, \zeta ; \sigma_{0}, l\right)=\pi^{*}\left(x, \zeta ; \sigma_{0}, l^{*}\right)$, where the asterisk denotes complex conjugate, and that $\pi(x$, $\left.\zeta ; \sigma_{0}, l\right)=\pi\left(x, \zeta ;-\sigma_{0},-l\right)$, so that the dispersion space is symmetric about the $\left(\sigma_{0}, l_{r}\right)$ plane, and also about the origin; that is, the dispersion surface $\sigma_{0}$ $=\sigma_{0 n}(l)$ for mode $n$ is reflected about the real plane and also through the origin. Therefore, for every growing wave, there is also a decaying wave whose structure function is the complex conjugate of that of the growing wave. We can consider only positive values of $l_{r}$ and negative values of $l_{i}$ without loss of generality.

In light of the above considerations, the eigenvalue search is carried out in the octant of dispersion space defined by $-0.6<\sigma_{0}<0,0 \leqslant l_{r}<10,-3.0 \leqslant l_{i}$ $<0$. For the range of parameters considered, $(2.8)$ is everywhere elliptic.

For a particular $\left(\sigma_{0}, l\right)$ pair, the system (2.8)-(2.9) is solved numerically on a finite difference mesh with 100 points in the $x$-direction and 40 points in the $\zeta$ direction. A direct elliptic solver (Adams, 1979) which takes advantage of the block-tridiagonal structure of the finite difference coefficient matrix is used to solve for the real and imaginary parts (2.8)-(2.9). The solver is extremely efficient, employing the hardware vector processing capabilities of the CRAY-1 computer.

\section{Background configuration}

The background density and velocity fields used in this study preserve the major features of the mean Gulf Stream as it flows along the Carolina continental margin. A velocity section off Cape Fear; North Carolina, is shown in Fig. 3, after Richardson et al. (1969). To approximate this velocity distribution, an analytical form for the background fields is chosen similar to that used by Orlanski and Cox (1973).

The alongshore velocity is assumed to be in thermal wind balance with the temperature field, which is given by

$$
T(x, z)=T_{d}-T_{0}\left[3-2\left(\delta x_{b}+1\right) e^{-\delta x_{b}}\right](\epsilon z-1) e^{2 \epsilon} z,
$$

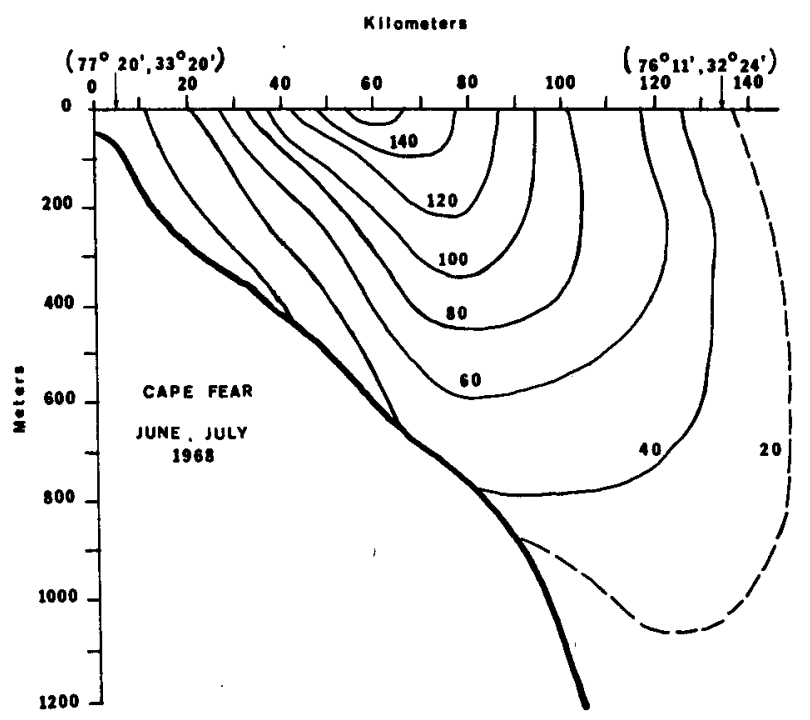

Fig. 3. Averaged velocity section of the Gulf Stream off Cape Fear, North Carolina, as determined from dropsonde data collected in June and July 1968 (from Richardson et al., 1969). Contours are isotachs of velocity into the page; interval is $20 \mathrm{~cm} \mathrm{~s}^{1}$. 
where $x_{b}=x-x_{f}$ if $x \geqslant x_{f}, x_{b}=0$ if $x<x_{f}$, where $x_{f}$ is the offshore position of the surface thermal front; $T_{d}$ is the deep water temperature, $T_{0}$ a reference temperature, $\delta$ the inverse of the horizontal length scale of the temperature field and $\epsilon$ is the inverse of the vertical length scale. The density is determined entirely from the temperature field:

$$
\rho(x, z)=\rho_{0}[1-a T(x, z)],
$$

where $a$ is the thermal expansivity of sea water and is taken to be a constant.

The Brunt-Väisälä frequency $N^{2}$ and its horizontal analog $M^{2}$ are then

$$
N^{2}(x, z)=-g a T_{0}\left[3-2\left(\delta x_{b}+1\right) e^{-\delta x_{b}}\right](2 \epsilon z-1) \epsilon e^{2 \epsilon} z,
$$

$$
M^{2}(x, z)=-2 g a T_{0} \delta^{2} x_{b} e^{-\delta x_{b}}(\epsilon z-1) e^{2 \epsilon} z .
$$

The background velocity is found by substituting (3.4) into (2.4) and integrating to get

$$
V(x, z)=\frac{g a T_{0}}{f_{\epsilon}} \delta^{2} x_{b} e^{-\delta x_{b}}\left(\frac{3}{2}-\epsilon z\right) e^{2 \epsilon} z+V_{I}(x) .
$$

Here, the integration constant $V_{I}(x)$ is a function of $x$ only, and for the purpose of modeling the Gulf Stream, will be taken to be zero. The horizontal shear is then

$$
\begin{aligned}
V_{x}(x, z) & =\frac{g a T_{0}}{f_{\epsilon}} \delta^{2}\left(1-\delta x_{b}\right) e^{-\delta x_{b}}\left(\frac{3}{2}-\epsilon z\right) e^{2 \epsilon} z \\
& =0 \begin{array}{ll}
\text { if } \quad x<x_{f} . & \text { if } x \geqslant x_{f}
\end{array}
\end{aligned}
$$

The maximum values of $N^{2}, M^{2}$ and $V$ are

$$
\begin{aligned}
N_{0}^{2} & =g a T_{0} \epsilon, \\
M_{0}^{2} & =2 g a T_{0} \delta e^{-1}, \\
V_{0} & =\frac{3 g a T_{0} \delta e^{-1}}{2 f_{\epsilon}} .
\end{aligned}
$$

The volume transport of the background flow, in the absence of bottom topography, is

$$
\text { Transport }=\frac{g a T_{0}}{f \epsilon} .
$$

The values for the parameters $a, T_{0}, \delta$ and $\epsilon$ are chosen so that the vertical and horizontal shear and the transport of the background velocity field closely resemble those of Fig. 3. Plots of the background fields are shown in Fig. 4.

The bottom topography has a hyperbolic tangent form:

$$
h(x)=H_{w}+\frac{1}{2}\left(H-H_{w}\right)\left\{1+\tanh \left[d_{1}\left(x-d_{2}\right)\right]\right\},
$$
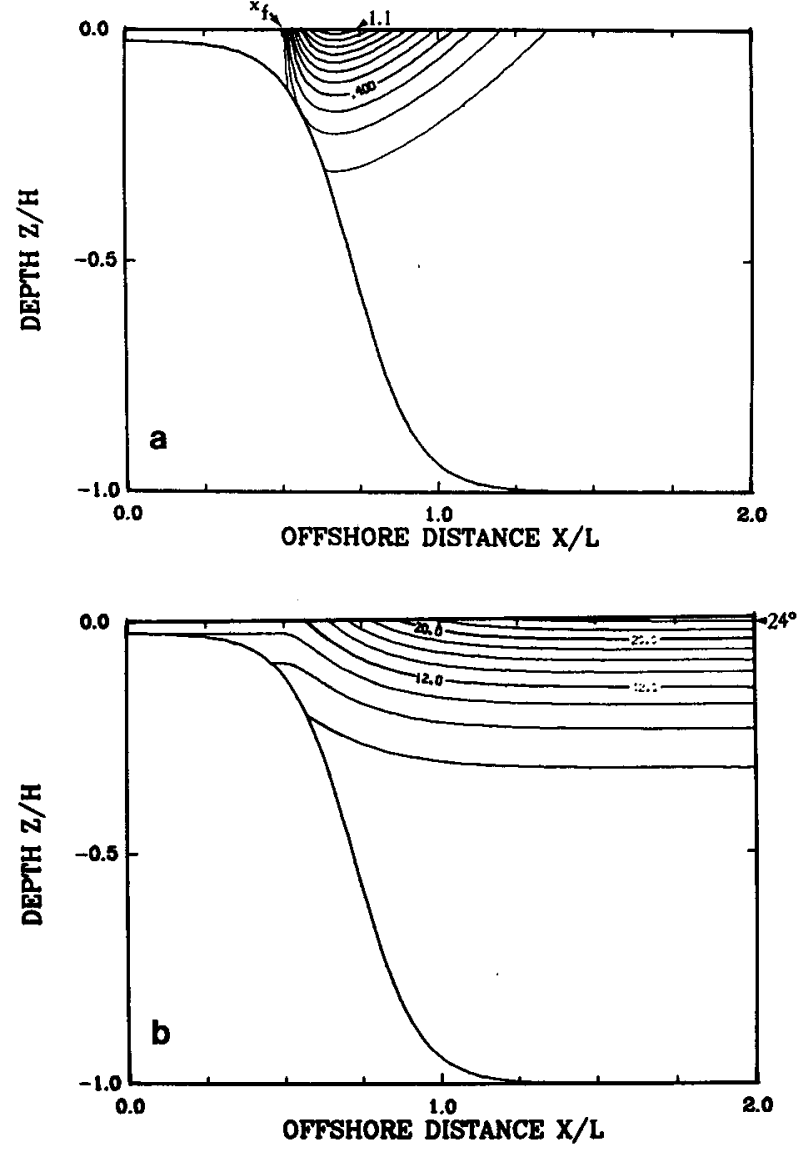

FIG. 4. Model fields. (a) Background (mean) velocity section. Contours are isotachs of velocity into the page in $\mathrm{m} \mathrm{s}^{-1}$, interval $0.1 \mathrm{~m} \mathrm{~s}^{-1} . x_{f}$ marks position of inshore surface front. (b) Background temperature field. Contours are in ${ }^{\circ} \mathrm{C}$, interval $2^{\circ} \mathrm{C}$.

where $H_{w}$ is the depth at the coast $(x=0), d_{1}$ is proportional to the maximum bottom slope and $d_{2}$ is the offshore position of the maximum bottom slope. The parameters $d_{1}$ and $d_{2}$ are chosen to approximate the continental shelf/slope topography off Onslow Bay.

The effect of different background fields and topographic configurations on the normal modes can easily be investigated by a systematic variation of the parameters $a, T_{0}, \delta, x_{f}, \epsilon, d_{1}$ and $d_{2}$; however, only one case will be presented here. The values of the parameters used in the following computations are listed in Table 1.

\section{Energy conversion}

Since the background flow possesses both vertical and horizontal shear, both the kinetic and potential energy of the background state are available as energy sources for the perturbations. Equations for the time rate of change of kinetic and potential energy for the perturbations can be formed from the dimensionless 
TABLE 1. Parameter values for background configuration.

\begin{tabular}{|c|c|}
\hline $\begin{aligned} a & =1.15 \times 10^{-4}{ }^{\circ} \mathrm{C}^{-1} \\
T_{0} & =7.0^{\circ} \mathrm{C} \\
\epsilon & =1.3 \times 10^{-3} \mathrm{~m}^{-1} \\
T_{d} & =4.0^{\circ} \mathrm{C} \\
\delta & =3.0 \times 10^{-5} \mathrm{~m}^{-1} \\
V_{0} & =1.25 \mathrm{~m} \mathrm{~s}^{-1} \\
\text { Transport } & =55 \times 10^{6} \mathrm{~m}^{3} \mathrm{~s}^{-1}\end{aligned}$ & $\begin{aligned} x_{f} & =100 \mathrm{~km} \\
d_{1} & =2.5 \times 10^{-5} \mathrm{~m}^{-1} \\
d_{2} & =145 \mathrm{~km} \\
H_{w} & =100 \mathrm{~m}\end{aligned}$ \\
\hline
\end{tabular}

form of (2.1) (a), (b) and (e). The perturbation kinetic energy is governed by

$$
\frac{1}{2} \frac{D}{D t}\left(\overline{u^{2}+v^{2}}\right)=-\left(\overline{u \pi_{x}}+\overline{v \pi_{y}}\right)-R_{0}\left(\overline{u v} V_{x}+\overline{v w} V_{z}\right) .
$$

The perturbation buoyancy variance is governed by

$$
\frac{D}{D t}\left(\frac{\overline{b^{2}}}{2}\right)=-\left(\alpha S^{2} M^{2} \overline{u b}+S^{2} N^{2} \overline{w b}\right) .
$$

Here, overbars denote an average over time. Equations (4.1) and (4.2) can be combined using (2.1c) to give an equation for the total perturbation energy:

$$
\begin{gathered}
\frac{1}{2} \frac{D}{D t}\left[\left(\overline{u^{2}+v^{2}}\right)+\frac{\overline{b^{2}}}{S^{2} N^{2}}\right)=-\left(\overline{u \pi_{x}}+\overline{v \pi_{y}}+\overline{w \pi} \bar{\pi}_{z}\right) \\
-R_{0}\left(\overline{u v} V_{x}+\overline{v w} V_{z}\right)-\alpha \overline{u b} \frac{M^{2}}{N^{2}} .
\end{gathered}
$$

The first term on the right-hand side of (4.3) is the pressure work term and represents a redistribution of perturbation energy. The second term represents the conversion of background kinetic energy to (from) perturbation energy, while the last term represents conversion of background potential energy to (from) perturbation energy. The magnitude of each of these terms can be readily computed for a particular value of $\left(\sigma_{0}, l\right)$.

If we again make the substitution, as in Section 2,

$$
(u, v, w, b)=\operatorname{Re}\left[\left(u^{\prime}, v^{\prime}, w^{\prime}, b^{\prime}\right) e^{i\left(\sigma_{0} t+l_{y}\right)}\right]
$$

the kinetic energy conversion term (KEC), or barotropic conversion term, becomes

$$
\begin{aligned}
\mathrm{KEC} & =-R_{0}\left(\overline{u v} V_{x}+\overline{v w} V_{z}\right) \\
& =\frac{1}{2} R_{0} e^{-2 l i y} \operatorname{Re}\left(u^{\prime} v^{\prime *} V_{x}+v^{\prime} w^{\prime *} V_{z}\right)
\end{aligned}
$$

and the potential energy, or baroclinic, conversion term (PEC) becomes

$$
\operatorname{PEC}=-\alpha \frac{M^{2}}{N^{2}} \overline{u b}=-\frac{\alpha M^{2}}{2 N^{2}} e^{-2 l i y} \operatorname{Re}\left(u^{\prime} b^{\prime *}\right) .
$$

These terms can be easily computed from the solutions to (2.8) and (2.9) using (2.7) and (2.1e). By integrating each of these terms over the $(x, z)$ plane and comparing the results, the instability mechanism for a particular wave may be classified as barotropic, baroclinic or mixed.

\section{Results}

The system (2.8)-(2.9) is forced at the top boundary with $\tau$ constant. For each value of $l$ the frequency $\sigma_{0}$ is varied from -0.01 to -0.6 , nominally in increments of 0.01 , and the response is plotted. Salby (1981) solved the same set of equations at different frequencies and wavenumbers for atmospheric Rossby waves in nonuniform background flows. He used a different numerical solver, and obtained very similar results to those presented here, which gives us confidence in our results. Figure 5.1 and 5.2 show the response for various values of wavenumber. Several peaks indicating a resonance response are clearly evident. Four general mode structures can identify most of the peak responses; however, the resonance responses corresponding to the waves with the lowest phase speeds could not be associated with a normal mode structure. Varying the spatial resolution of the numerical method produced large variations in the spatial structure of these waves. This is believed to be the result of critical level effects, since these low phase speed waves have critical levels that intersect the bottom topography nearly tangentially. The equations of motion become singular where the wave-phase velocity is cancelled by the background flow velocity, i.e., at the critical level, unless the growth rate, $-l_{i}$, is nonzero. The effect of a nonzero growth rate mathematically is to change the critical level from a singularity where discontinuities in the solution can occur into a thin layer where strong gradients in the solution are possible. In this sense, the imaginary part of the wavenumber acts like viscous dissipation. In order to resolve the critical level numerically, there must be a sufficient number of solution mesh points within this layer. If the critical level intersects the topography at a low angle, the gradients in the solution near the intersection become so sharp that they are difficult to resolve numerically. The very low-frequency response peaks are therefore thought to be noise in the numerical method. All the numbered normal-mode structures are well defined and independent of decreasing mesh size.

All the normal mode structures are trapped near the surface and extend to the bottom only over the continental shelf and upper slope. The mode number $n$ assigned to each mode corresponds to the number of nodes in perturbation pressure along the surface for that mode. Each mode may have more than one response peak, indicating a splitting of the normalmode frequency due to nonuniformities in the background state. This is very similar to the spectral broadening and translation found by Salby (1981) in a similar study of atmospheric waves. He found that the "effective" background nonuniformity, and hence 
the influences which degrade the resonance response, increase with increasing real wavenumber and mode number. This can clearly be seen here by comparing Fig. 5a and $b$. The response for modes 0 and 1 remains sharp with increasing real wavenumber, while the response for mode 2 splits at high wavenumber and that for mode 3 is spread out over a wide band of frequencies, and for some values of $l_{r}$ is not even recognizable. The response peaks for 0,1 and 2 all begin to split as real wavenumber increases, although this is much more pronounced for mode 2. Again, this is due to the increased "effective" background nonuniformity for higher mode number. The resonance frequencies for all modes tend to shift in the negative direction with increasing $l_{r}$. A key feature to note in all of the response curves is the precipitous drop in the response for frequencies greater in magnitude than that at which the response for mode 0 occurs. At these frequencies, no normal modes can exist, and the only response is that driven directly by the wind stress curl. This directly driven response is the noise in energy spectrum, and any time the
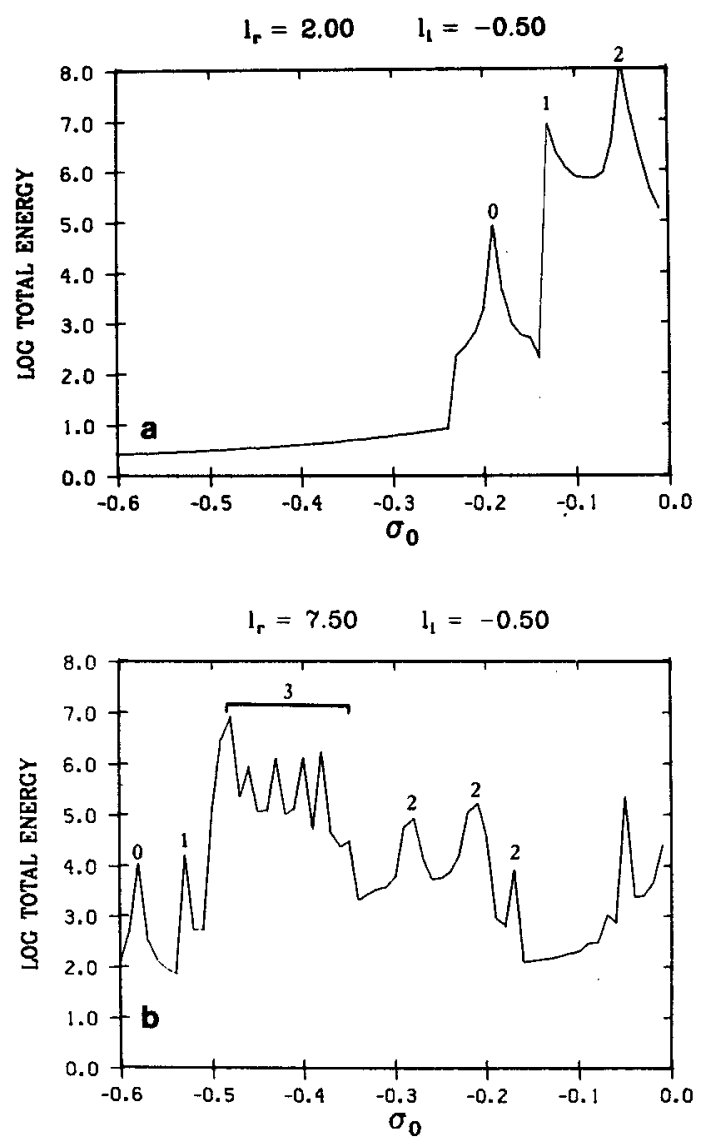

FIG. 5. Frequency response spectra for intermediate growth rate. $\log _{10}$ of the integrated total energy plotted against dimensionless frequency for (a) low real wavenumber and (b) high real wavenumber.
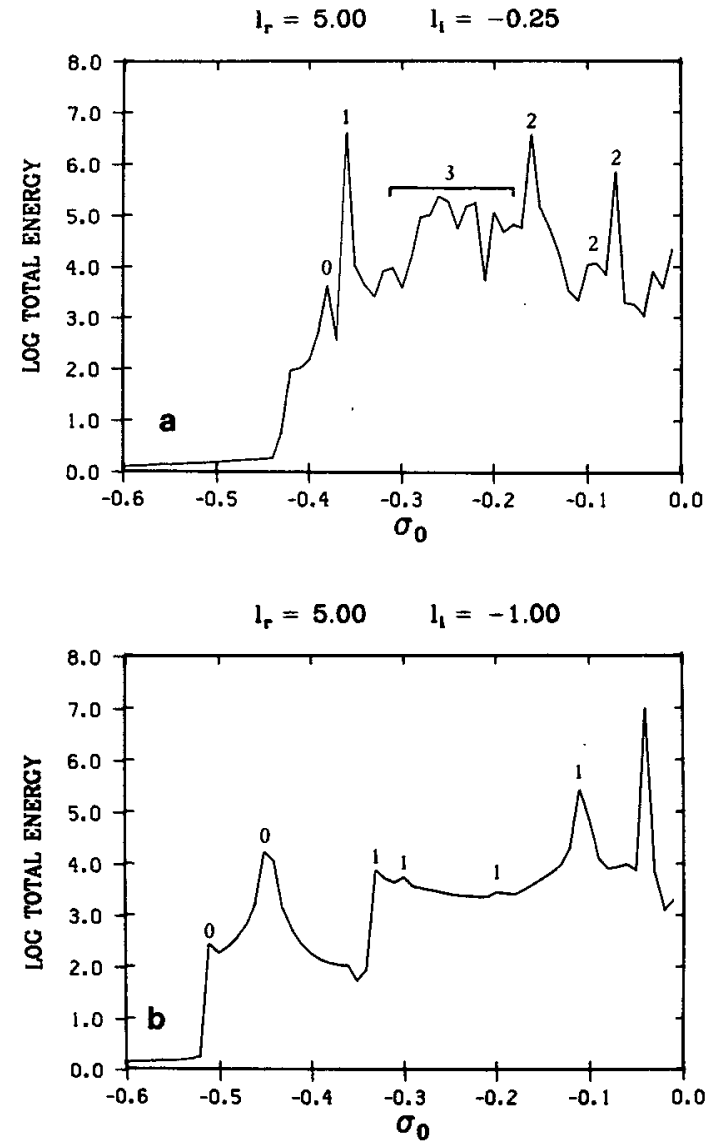

FIG. 6. As in Fig. 5 for intermediate real wavenumber and (a) low growth rate; (b) high growth rate.

response rises significantly above this noise level, it must be due to the presence of normal mode energy in the response.

The behavior of the response with increasing growth rate $\left|l_{i}\right|$ is somewhat ambiguous (see Fig. 6). The response for mode 3 is suppressed considerably with increasing growth rate, and at $l_{i}=-1.0$, is barely recognizable. Mode 2 shows a response that peaks at fewer frequencies as growth rate increases, while modes 0 and 1 tend to exhibit a response that peaks at a greater number of resonant frequencies. The frequencies of the resonance responses vary with increasing growth rate for all modes, especially mode 0 , which shows a shift to larger (more negative) frequencies. At high growth rates $\left(l_{i} \sim-4.0\right)$ the noise level in the response dominates the response, while peaks corresponding to mode 0 and possibly mode 1 are still discernible. At very low growth rates $\left(l_{i} \sim-0.05\right)$ the mode 2 and 3 responses are so diffused that they overlap considerably and essentially dominate the spectrum, so that no distinct normal mode structure is apparent.

The resonance frequencies for each mode at a 
particular value of $l$ can be interpreted as the eigenfrequencies for that mode at that wave number as described in Section 2. Dispersion relations for each mode can be determined by computing the response at many different values of $l$. The splitting of the resonance frequency for a particular mode is thus interpreted as a bifurcation of the dispersion surface in frequency-wavenumber space. The increase in the noise level at very high growth rates may be indicative of an upper bound on the growth rate for the perturbations, as it has been shown in quasi-geostrophic theory that the growth rate is bounded by the sum of the available background potential and kinetic energy (cf. Pedlosky, 1979, Section 7.5). The absence of any distinct normal-mode structure at low growth rates is due to the fact that no neutral waves can exist that have a critical level in the background flow (Bretherton, 1966a). Indeed, at very low growth rates, an essentially flat spectrum results.

There are several uncertainties in locating eigenfrequencies. Due to the computational expense of searching for the resonance responses, the increments in frequency and wavenumber were not as small as
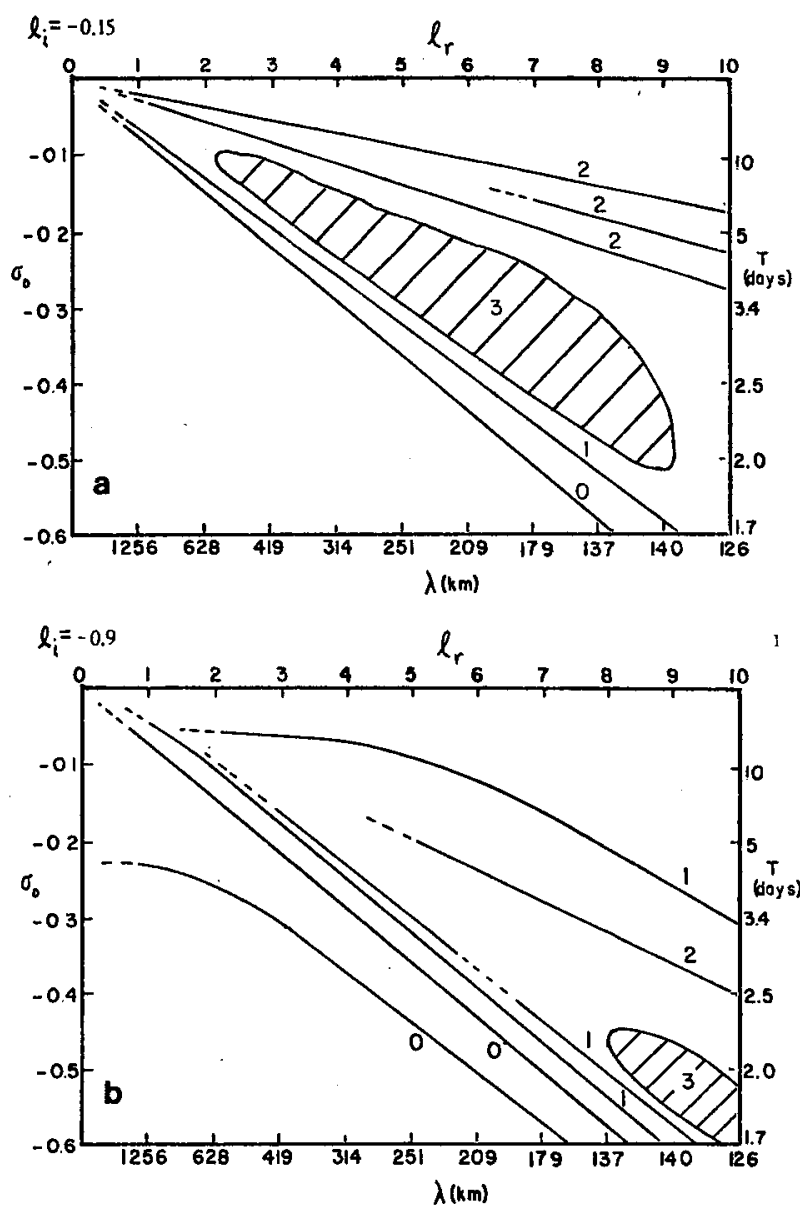

that which might have been desired. Some very narrow resonance peaks may therefore have been stepped over, and some broad peaks may actually be the banding of several very narrow peaks. For the same reason, the location of bifurcations in the dispersion surfaces can only be estimated. The wide bandwidth of some of the resonance responses introduces another uncertainty in the location of the eigenvalues. Indeed, in some instances, especially for mode 3 , there is no single peak frequency, but a range of frequencies over which there is an enhanced response. With these uncertainties in mind, we will present the dispersion surfaces.

It is somewhat difficult to present a two dimensional representation of a complicated three-dimensional surface. The occurrence of multiple bifurcations in these surfaces only adds to the difficulty. We resort here to presenting slices through dispersion space for constant values of either $l_{r}$ or $l_{i}$. These cross sections of the dispersion surfaces are shown in Fig. 7. Dashed lines represent areas of uncertainty in location of the surface, while cross-hatched areas represent frequency bands where a general enhanced response with no
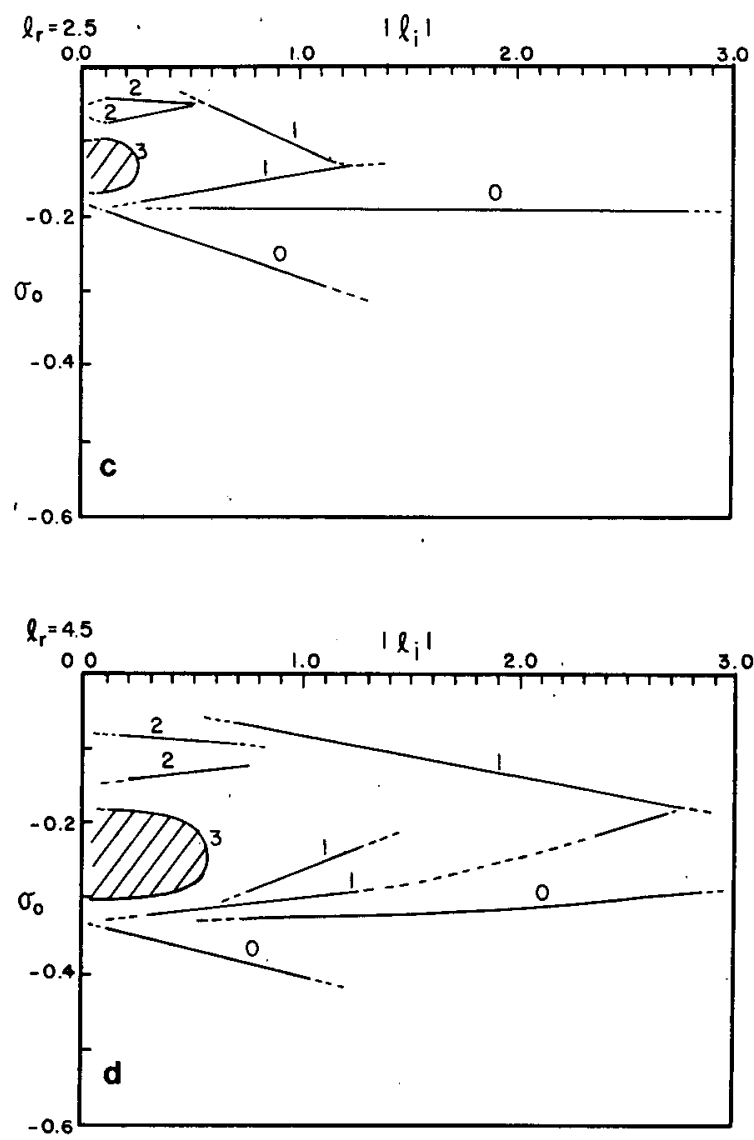

FIG. 7. Cross sections through dispersion space for (a) $l_{i}=-0.15$, (b) $l_{i}=-0.9$, (c) $l_{r}=2.5$ and (d) $l_{r}=4.5$. 
dominant peaks was found. The general features of the resonance responses described above are clearly seen in the dispersion diagrams. All modes appear to be essentially nondispersive at low growth rates, with phase speeds varying only slightly with growth rate. The upper branch for mode 0 and the lower branch for mode 1 become dispersive at low real wavenumber for higher growth rates. Modes 0 and 1 exhibit bifurcations at high growth rate and low wavenumber, while mode 2 tends to bifurcate at lower growth rate and higher real wavenumber. The volume of enhanced response for mode 3 tends toward higher frequency and real wavenumber as growth rate increases. Modes 0 and 1 appear to have the highest growth rates, while modes 2 and 3 have much lower growth rates; however, due to the limitations of the resonance search, the upper bound on the growth rate for each mode is uncertain.

We will describe the normal mode structures by presenting the cross-stream amplitude and phase of the pressure and velocity components. A problem encountered in presenting these structures is that the phase of the complex structure function undergoes a $180^{\circ}$ jump across nodes in amplitude. Where the amplitude is very near zero, the phase can become quite erratic, and has essentially no significance.

The cross-stream structure for mode 0 at moderate real wavenumber is shown in Fig. 8 . This wave has no nodes in amplitude and its phase is essentially constant over the region where the amplitude is significant. Its amplitude is concentrated in the background current and has a maximum at the core of the background current. There are two branches of the mode 0 dispersion surface in Fig. 7. The lower branch (the branch corresponding to lower $\left|\sigma_{0}\right|$ ) has a phase speed equal to the maximum background current speed. It therefore has a degenerate critical level along the surface axis of the background current; that is, the mode 0 wave has its frequency Doppler shifted to zero along a line at the surface following the core of the background current. The upper branch of the mode 0 dispersion surface has a phase speed which increases with growth rate. Its phase speed is always slightly greater than the maximum current speed, so it does not have a critical level. At moderate to high values of $l_{r}$, its cross-stream structure is identical to that of Fig. 8; however, at low values of $l_{r}$, this wave has a large component over the shelf. The amplitude of this shelf component increases with increasing growth rate. It is not clear whether this wave is truly an eigenmode of the system, since it travels faster than the background current. The resonance peaks for this branch are considerably smaller than those for the lower branch, as can be seen in Fig. 6b. It is possible that this is a topographic effect, with the sloping topography acting to destabilize the flow, but the bottom slope appears to act in the wrong sense.
The energy conversion mechanism is a mixed barotropic-baroclinic instability. A measure of the relative importance of baroclinic instability to barotropic instability is obtained by integrating (4.4) and (4.5) and taking their ratio:

$$
\mathrm{TPE} / \mathrm{TKE}=\int_{0}^{L} \int_{-h}^{0} \mathrm{PEC} d z d x / \int_{0}^{L} \int_{-h}^{0} \mathrm{KEC} d z d x .
$$

For the lower branch of mode 0 , and for the upper branch at moderate to high values of $l_{r}$, this ratio ranges from 4 to 5 , while for waves with a large shelf amplitude, it can approach unity. The potential energy conversion for all mode 0 waves takes place in the center of the background current, the region of maximum isopycnal slope, while the kinetic energy conversion occurs in the frontal zone, where the maximum horizontal current shear is found. The kinetic energy conversion due to the horizontal shear of the background current [the first term on the right-hand side of (4.4)] is everywhere an order of magnitude greater than the kinetic energy conversion due to the vertical shear of the background current [the second term in (4.4)] for mode 0 as well as for the other modes identified.

There are several general features of the velocity components that are common to all modes. The $u$ and $w$ components are nearly $90^{\circ}$ out of phase with $\pi$, except in the regions where energy conversion occurs. The $v$ component always has one more node than $\pi$, and is either in phase or $180^{\circ}$ out of phase with $\pi$. The phase for the pressure and velocity components is nearly constant with depth, again except in the areas of energy conversion. It is important to note that the energy conversion takes place in very limited areas, implying that energy conversion estimates from field measurements may be misleading due to their limited spatial resolution.

Mode 1 has a single pressure node at the center of the background current, with the maximum amplitude in each of the two lobes centered on the intersection of the wave's critical level with the surface (Fig. 9). The phase makes a $180^{\circ}$ jump across the node, but is nearly constant everywhere else. As with mode 0 , the dispersion surface for this wave bifurcates at high growth rates and low values of $l_{r}$. The amplitude of the mode 1 wave is confined to the background current, with very little amplitude away from the current. The instability mechanism is primarily baroclinic, with TPE/TKE ranging from 10 to 15; however, both PEC and KEC are negative in some areas, indicating a slight return of wave energy to the background flow. Most of the energy conversion occurs in the region bounded by the wave's critical level and the surface boundary. The wave amplitude appears to become confined to smaller areas about the intersections of the critical level with the surface as $\left|\sigma_{0}\right|$ increases and $\left|l_{i}\right|$ decreases. 

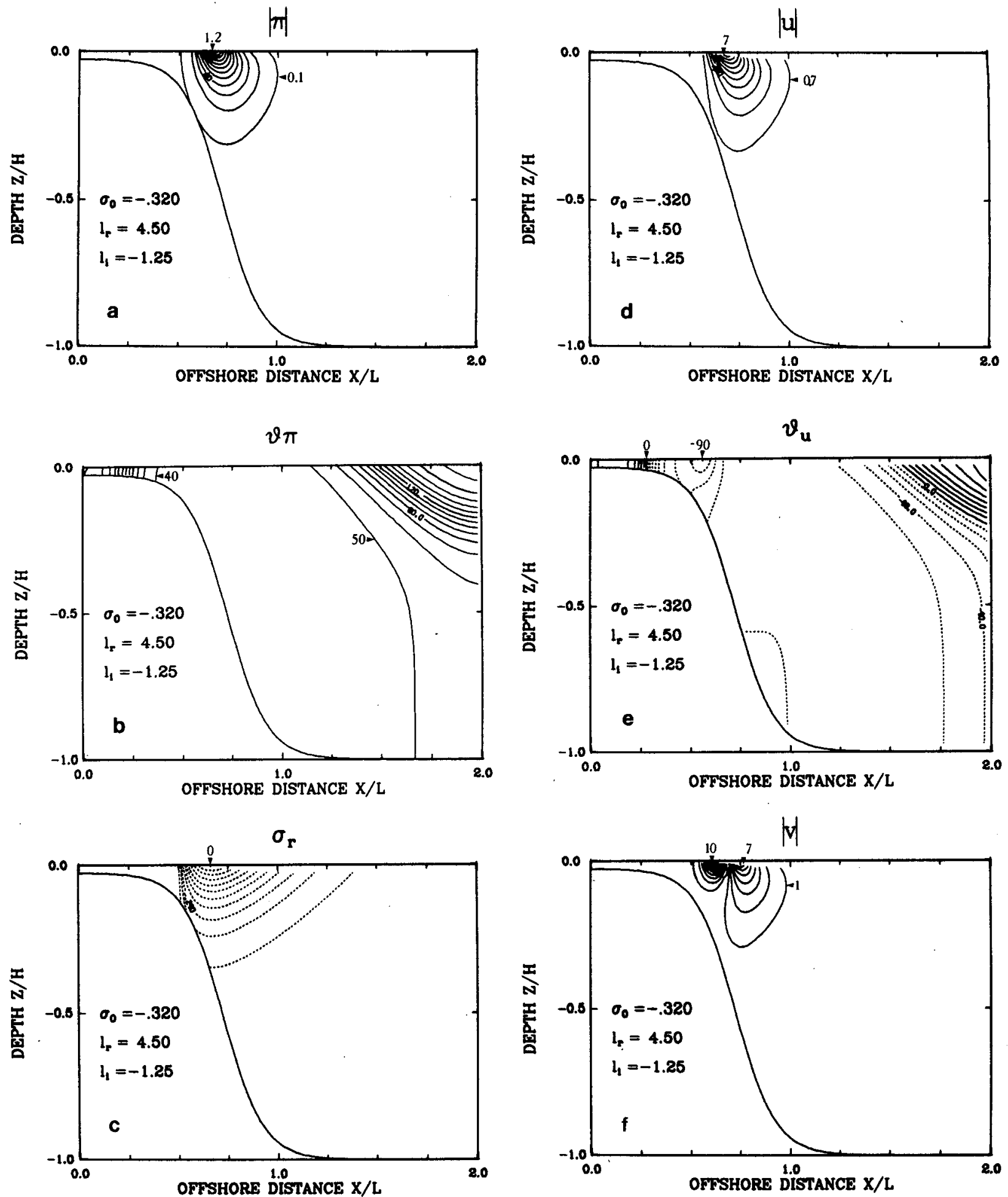

FIG. 8. Normal-mode structure functions for mode 0 at the lower phase speed dispersion surface. Velocity components are not computed along boundaries to avoid making one-sided difference approximations. (a) Perturbation pressure $\pi$, amplitude in arbitrary units, contour interval 0.1. (b) Phase of $\pi$ in degrees, contouor interval $10^{\circ}$. (c) Normalized intrinsic (Doppler-shifted) frequency $\sigma_{r}$ for mode 0 , contour interval 0.03 . (d) Cross-shelf velocity amplitude in arbitrary units, contour interval 0.7 . (e) Phase of cross-shelf velocity in degrees. Contour interval $10^{\circ}$. (f) Alongshore velocity amplitude in arbitrary units, contour interval 1.0 . (g) Phase for alongshore velocity in degrees, contour interval $30^{\circ}$. (h) Vertical velocity amplitude in arbitrary units, contour interval 0.3. (i) Phase of vertical velocity in degrees, contour interval $30^{\circ}$. 

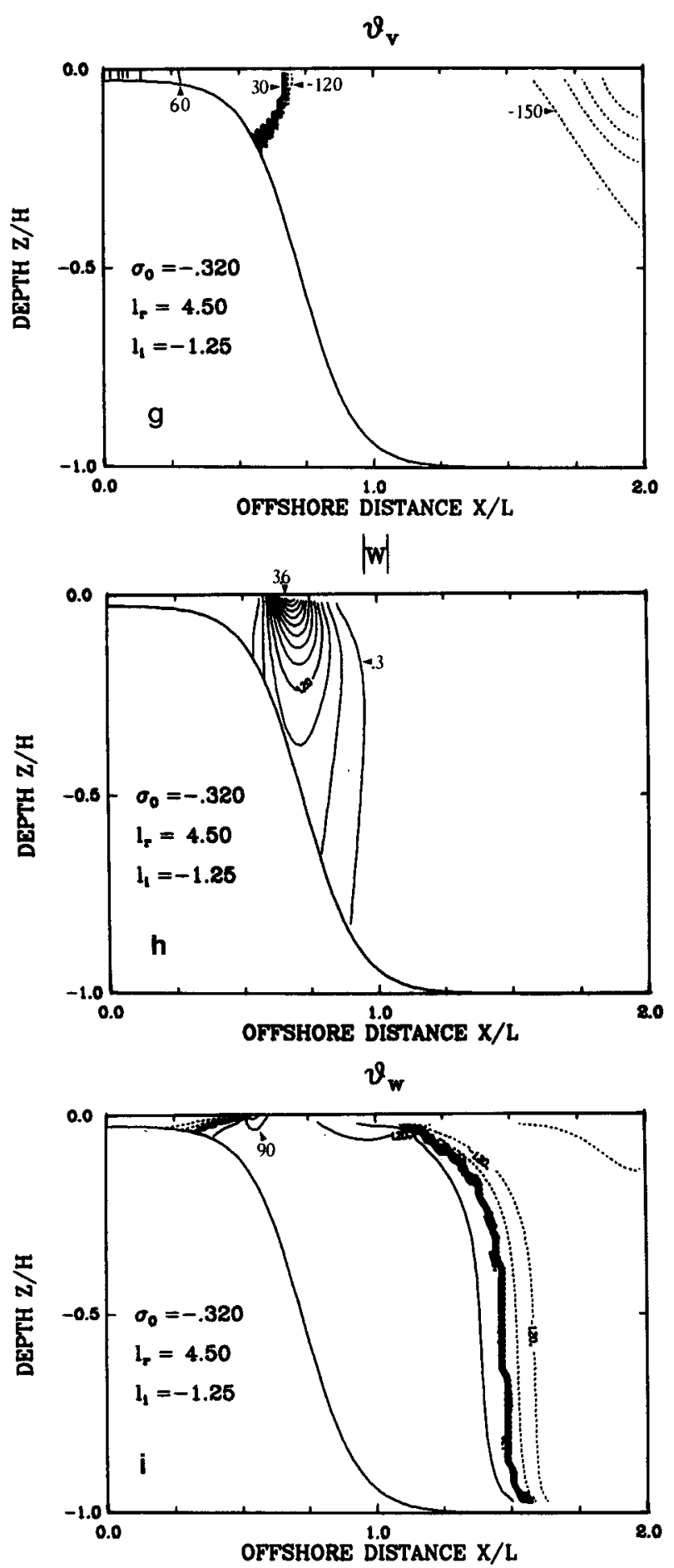

FIG. 8. (Continued)

The mode 2 wave structure is shown in Fig. 10. This wave has two nodes in perturbation pressure occurring between the two intersections of the wave's critical level with the surface. The two lobes in pressure amplitude on the outside of the nodes are centered about the critical level's intersections with the surface. The maximum amplitude occurs at the surface between the two nodes. Again, the amplitude is greatest in the background current and decays rapidly away from it. The phases of the pressure and velocity components again undergo $180^{\circ}$ phase shifts across the nodes and are essentially constant elsewhere. The energy conversion mechanism is a mixed baroclinic/barotropic instability, with TPE/TKE ranging from 5 to 6 . The potential energy conversion is everywhere from the background to the perturbation, while the kinetic energy conversion is both positive and negative, indicating a partial return of perturbation energy to the kinetic energy of the background flow. Then it appears that the mode 2 wave extracts energy from the background by eroding the horizontal temperature gradient, while returning energy to the background by increasing the horizontal shear. The potential energy conversion occurs in the core of the background current in the area bounded by the critical level and the surface boundary. The kinetic energy conversion occurs in the cyclonic frontal zone on either side of the inshore critical level intersection.

The phase speed for the largest mode 2 response is constant with wavenumber and is equal to the average
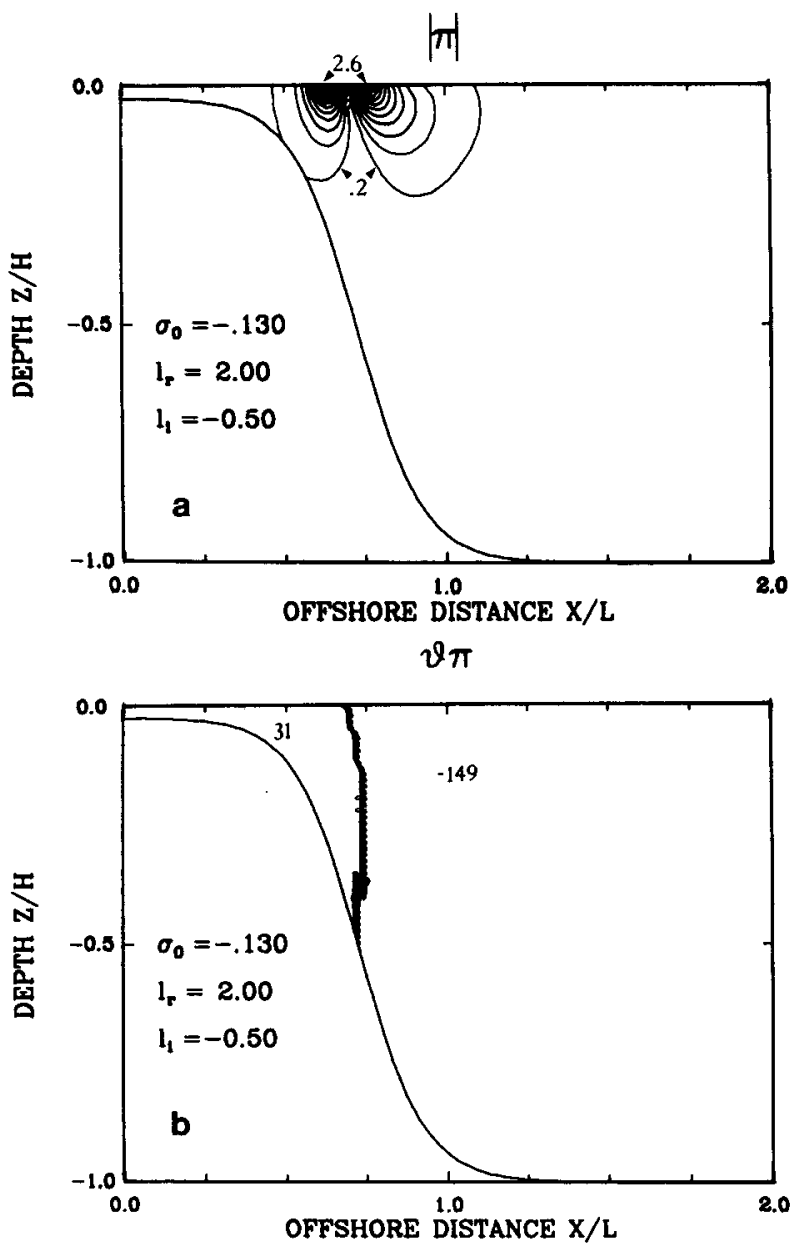

Fig. 9. Structure functions for mode 1: (a) amplitude for $\pi$, contour interval 0.2 ; (b) phase for $\pi$ in degrees, contour interval $10^{\circ}$. 

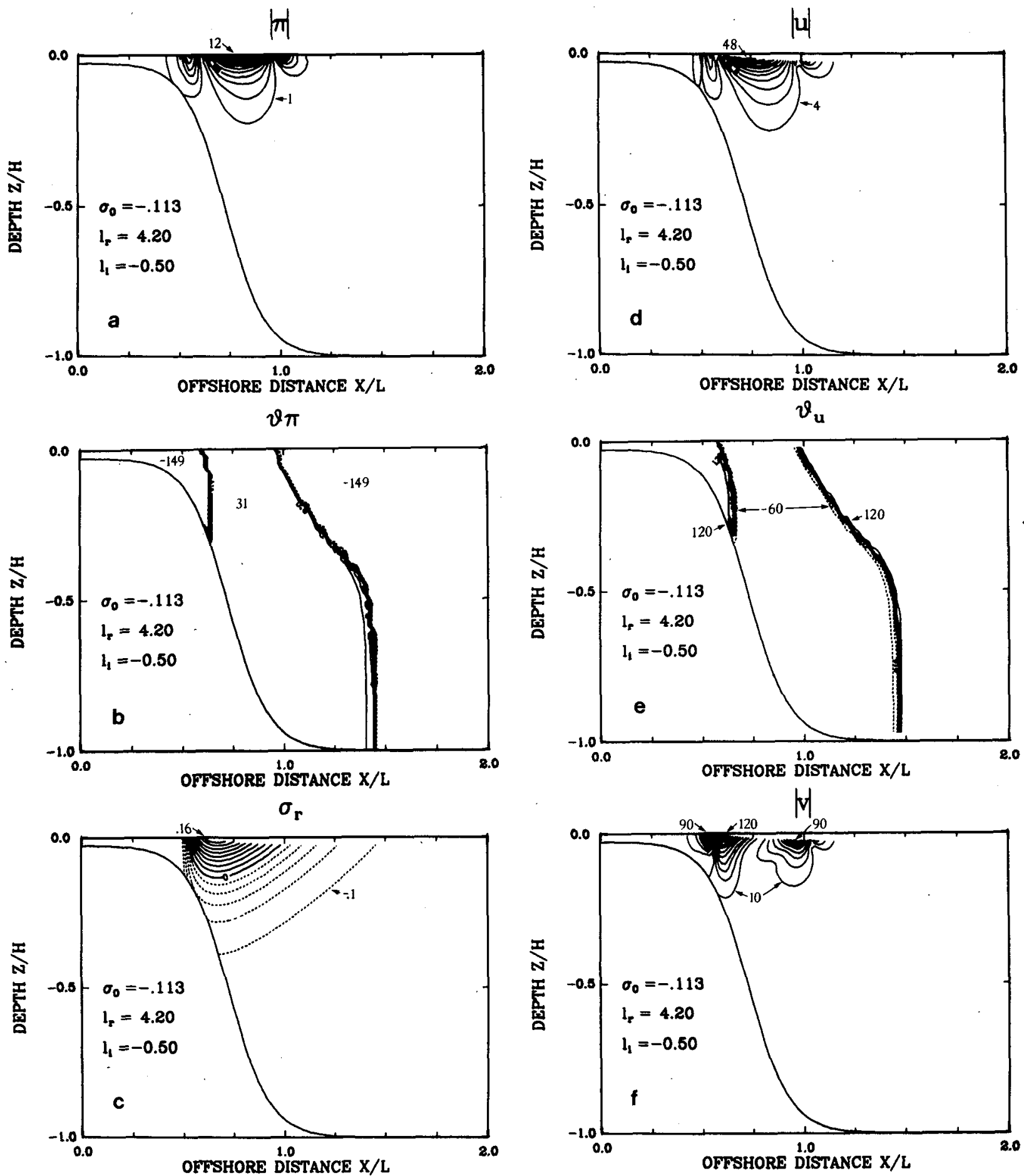

FiG. 10. Structure function for mode 2: (a) amplitude for $\pi$, contour interval 1.0; (b) phase for $\pi$, contour interval $20^{\circ}$; (c) intrinsic frequency $\sigma_{r}$, contour interval 0.02 ; (d) amplitude for $u$, contour interval 4.0 ; (e) phase for $u$, contour interval $30^{\circ}$; (f) amplitude for $v$, contour interval 10.0 ; (g) phase for $v$, contour interval is $30^{\circ}$; (h) amplitude for $w$, contour interval is 0.4 ; and (i) phase for $w$, contour interval $30^{\circ}$.

background current speed, about $40 \mathrm{~cm} \mathrm{~s}^{-1}$. This implies that the wave's critical level remains fixed for different values of $l$. It is interesting to note that the most unstable wave found by Eady (1949) has a phase speed equal to the average background current speed, as did the most unstable wave found by Orlanski and Cox (1973).

The structure of the mode 3 wave is shown in Fig. 

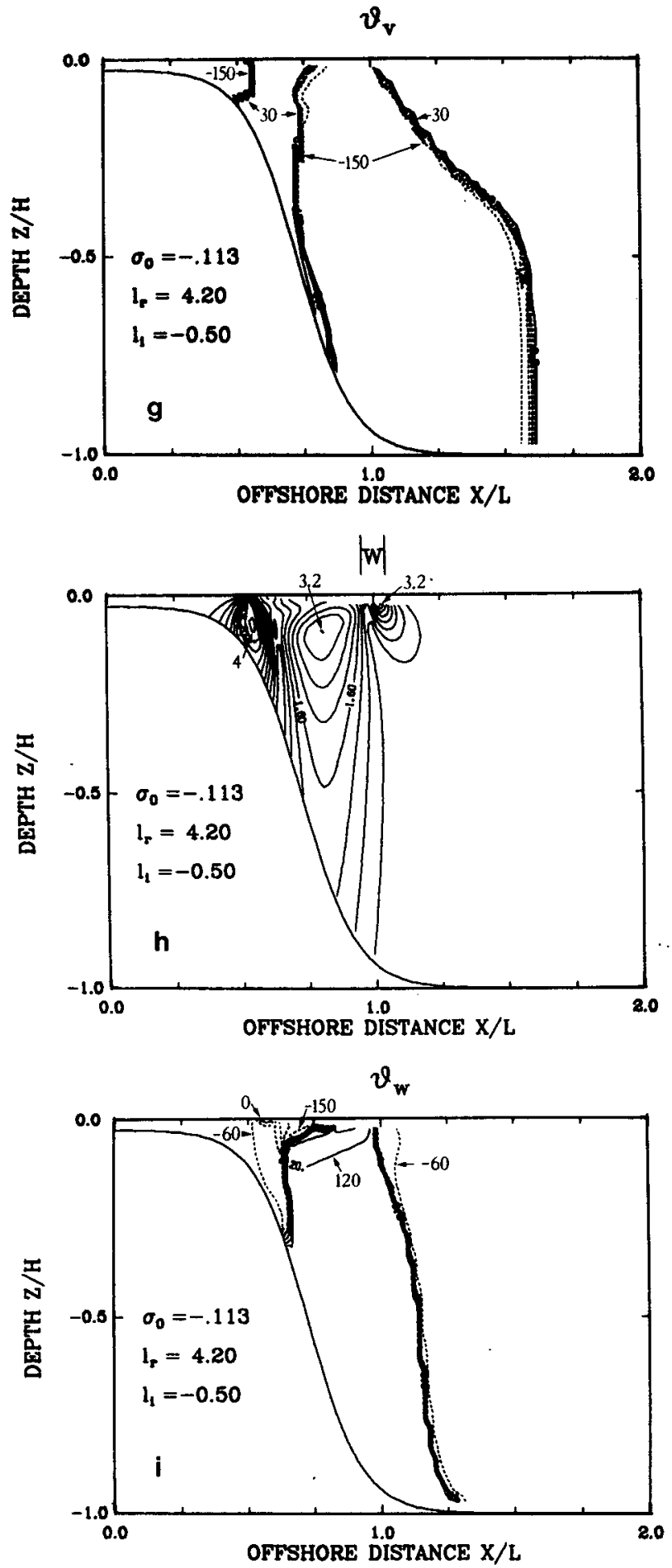

Fig. 10. (Continued)

11. It exhibits most of the general features described for the mode 1 wave. As mentioned above, the mode 3 response is so diffused that it is difficult to draw any conclusions about its eigenvalue relationships. It does appear that the mode 3 wave has a low growth rate and that the growth rate increases somewhat with increasing real wavenumber. The complexity of its spatial structure makes it difficult to resolve numerically. The kinetic energy conversion for this mode is usually from the perturbations to the background on the average, while the potential energy conversion is always positive, i.e., from the background to the perturbations. The ratio TPE/TKE ranges from -10 to -50 , indicating that this mode may be responsible for a redistribution of energy in the background flow.

\section{Comparison with observations}

The dimensionless frequency and real wavenumber for the observed 8-day wave are computed from current meter records from Onslow Bay, North Carolina (Brooks and Bane, 1981; Bane et al., 1981) to be -0.13 and 4.2 respectively. Assuming a moderate growth rate for the disturbance, the frequency and wavenumber for the 8-day wave lie right on the mode
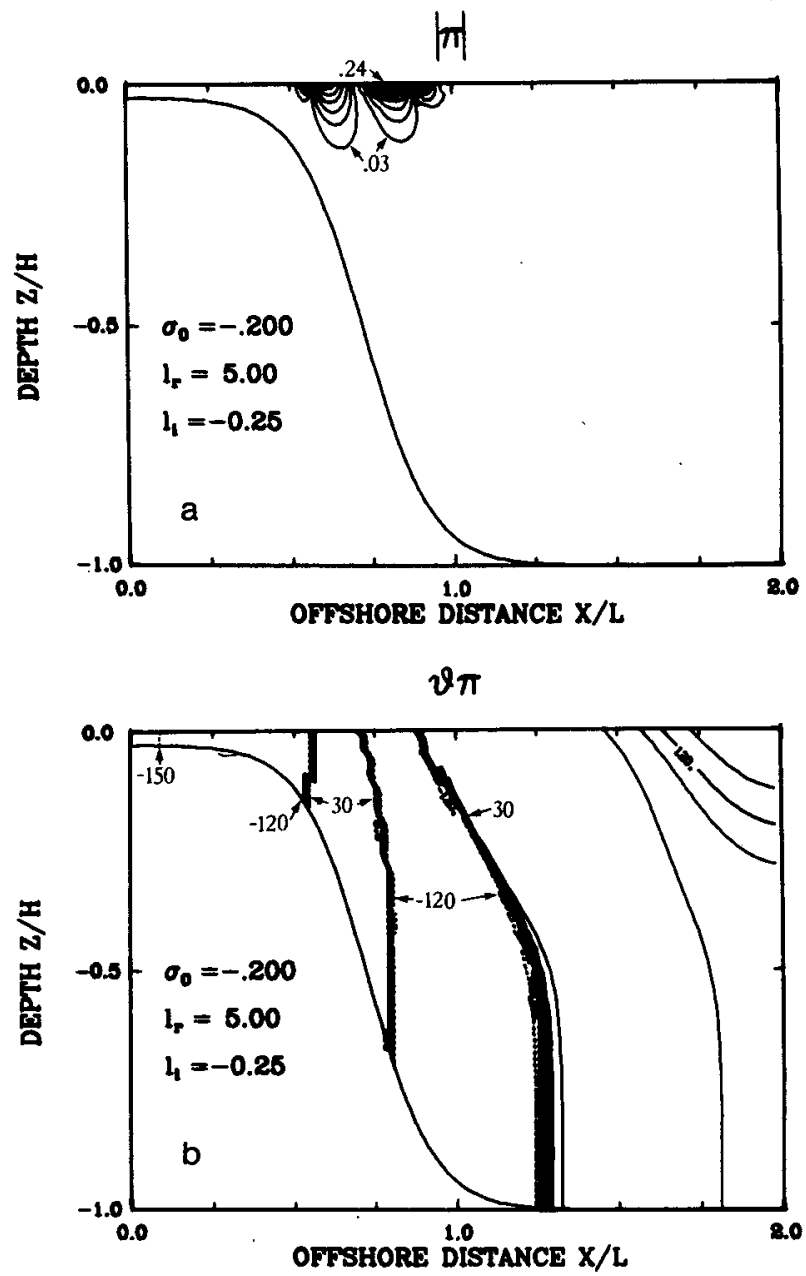

Fig. 11. Structure functions for mode 3: (a) amplitude for $\pi$, contour interval 0.03 . (b) Phase for $\pi$, contour interval $30^{\circ}$. 
2 dispersion surface. The structure of the mode 2 wave is consistent with that of the 8-day wave. The observed perturbation velocity has no nodes in the vertical, but decreases with depth and with distance away from the Stream. To illustrate the current patterns that would be associated with a mode 2 wave, if realized in finite amplitude, we have superimposed the perturbation velocity onto the background velocity, with an amplitude of $20 \%$ of the maximum background velocity, which is consistent with current meter measurements (Fig. 12). The resulting meander pattern consists of alternating cyclonic and anticyclonic eddies between the two perturbation pressure nodes, about which the core of the current meanders. Smaller counter-rotating eddies are found on the outside of the nodes. A meander trough (the seaward-most excursion of the core of the current) consists of a cyclonic eddy on the inshore side of the main current, with two smaller anticyclonic eddies on either side. Conversely, a meander crest (the shoreward-most excursion of the core of the current) consists of an anticyclonic eddy seaward of the main current, with two smaller cyclonic eddies on either side. The vertical velocity and perturbation buoyancy near the surface associated with this wave are shown in Figs. $12 \mathrm{~b}, \mathrm{c}$ respectively. In the trailing portion of a meander crest, there is upwelling between the nodes, just ahead and slightly offshore of the cyclonic eddy. Outside of the perturbation pressure nodes, there is downwelling in this part of the meander pattern. In the leading portion of a meander crest the situation is reversed, with downwelling inside the nodes and upwelling outside. Near the surface, the vertical velocity pattern is skewed, resembling the observed meander and warm filament structure of Fig. 1. This skewness means that $u$ and $w$ are not in phase and thus that $u$ and $b$ are not in quadrature from (2.1e), which in turn leads to potential energy conversion from the background to the perturbation in (4.5). The skewness in the $w$ field is most pronounced in the upper levels of the water column, and diminishes greatly at depth (Fig. 13b). Since a negative perturbation buoyancy indicates the presence of cooler water, we can see that there is a tendency for a dome of cooler water to form at the center of the cyclonic eddy, with upwelling on the leading side of the dome and downwelling on the trailing side. This is consistent with the conceptual model of the

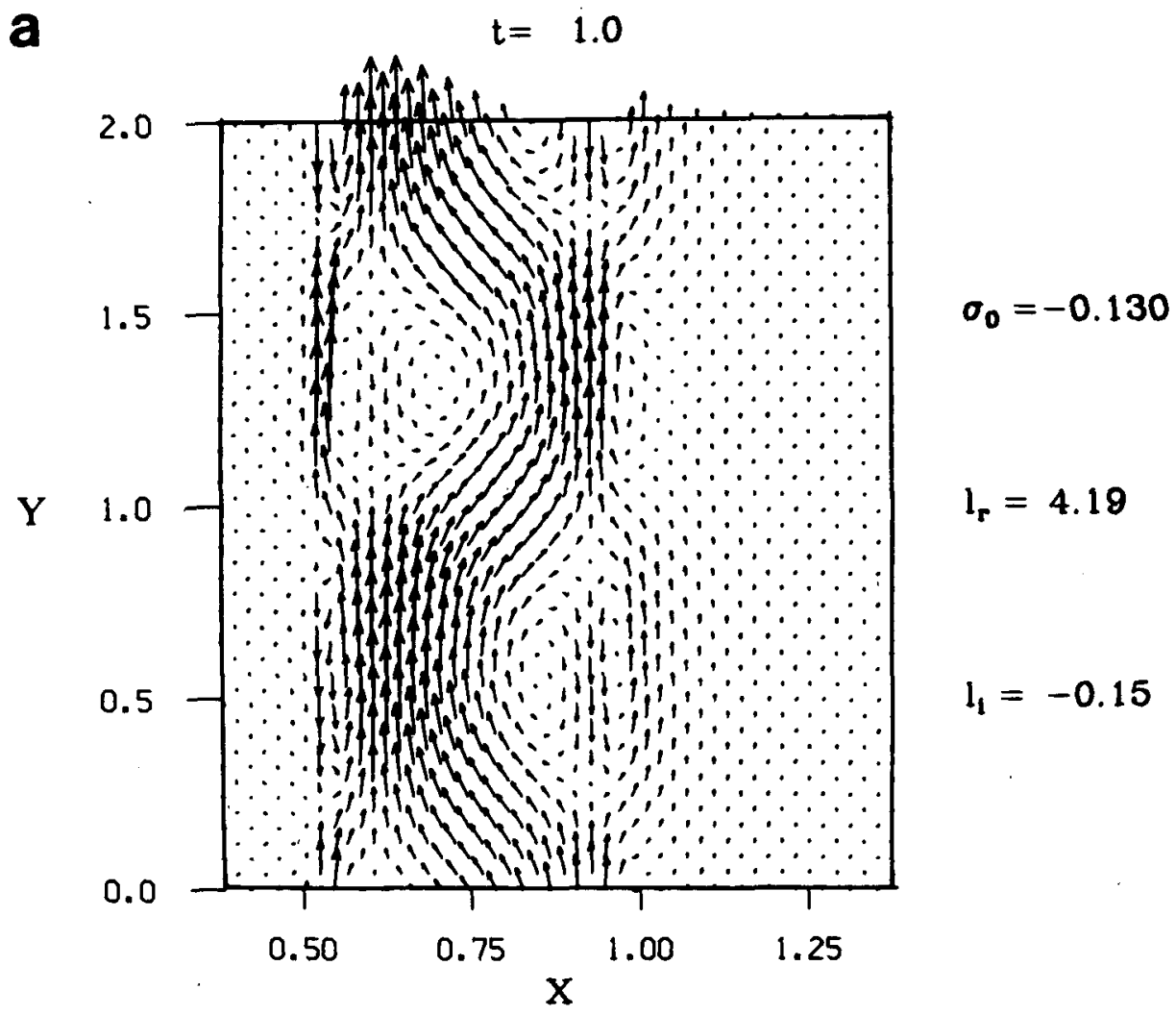

FIG. 12. (a) Surface currents for the mode 2 wave at the 8-day period superimposed on the background current. (b) Horizontal distribution of vertical velocity near the surface $(\zeta=-0.026)$ for the mode 2 wave at the 8-day period. (c) Horizontal distribution of buoyancy flux near the surface ( $\zeta$ $=-0.026$ ) for the mode 2 wave at the 8 -day period. 
b $t=1.0$

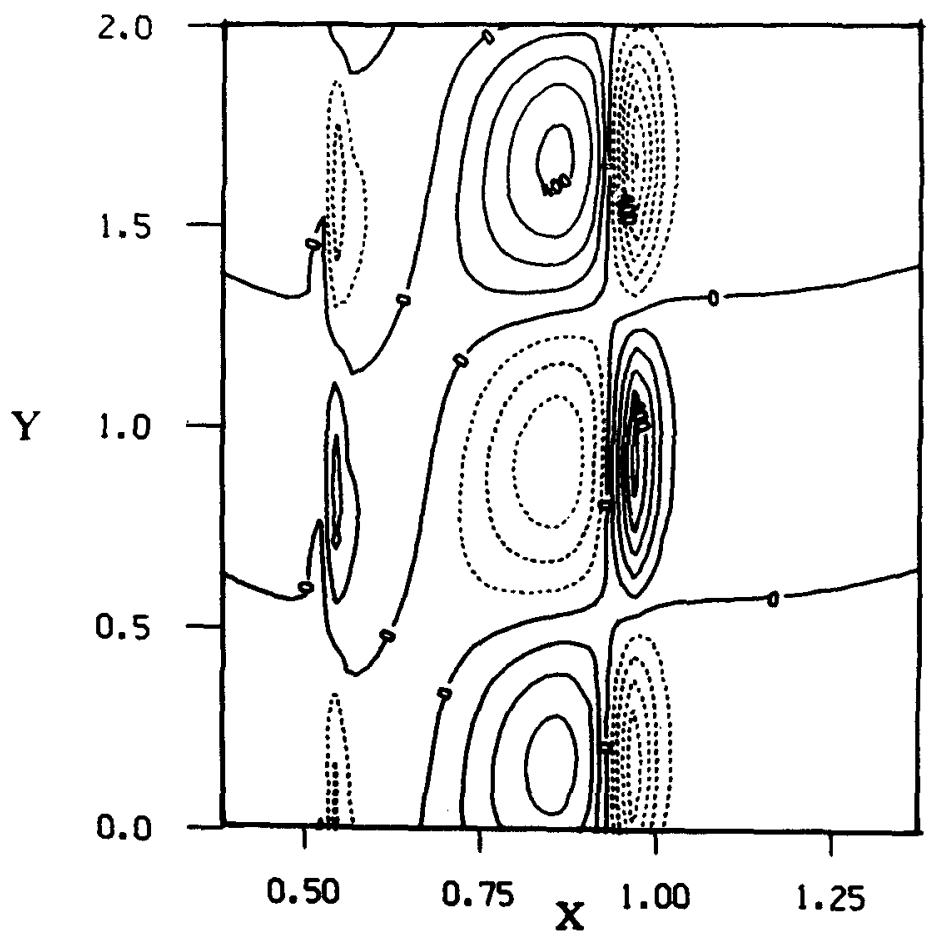

$$
\begin{aligned}
& \sigma_{0}=-0.130 \\
& l_{r}=4.19 \\
& l_{1}=-0.15
\end{aligned}
$$

C

$$
t=1.0
$$

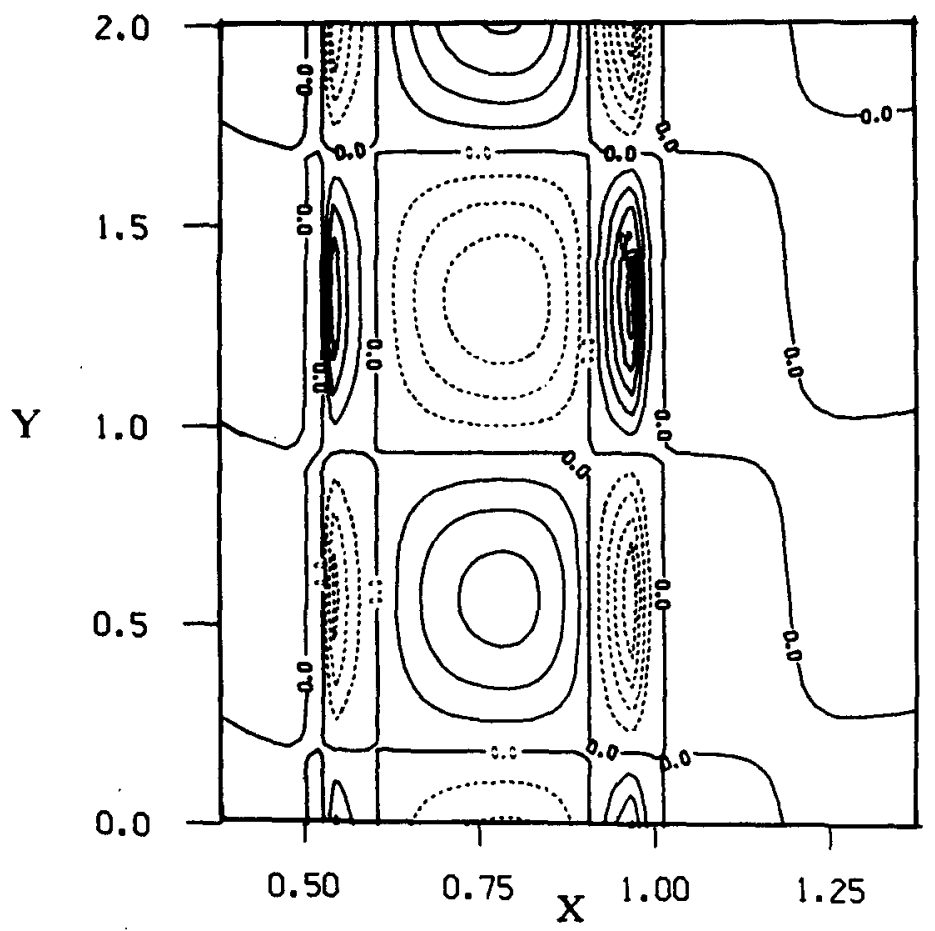

$\sigma_{0}=-0.130$

$l_{r}=4.19$

$l_{1}=-0.15$

FIG. 12. (Continued) 
a

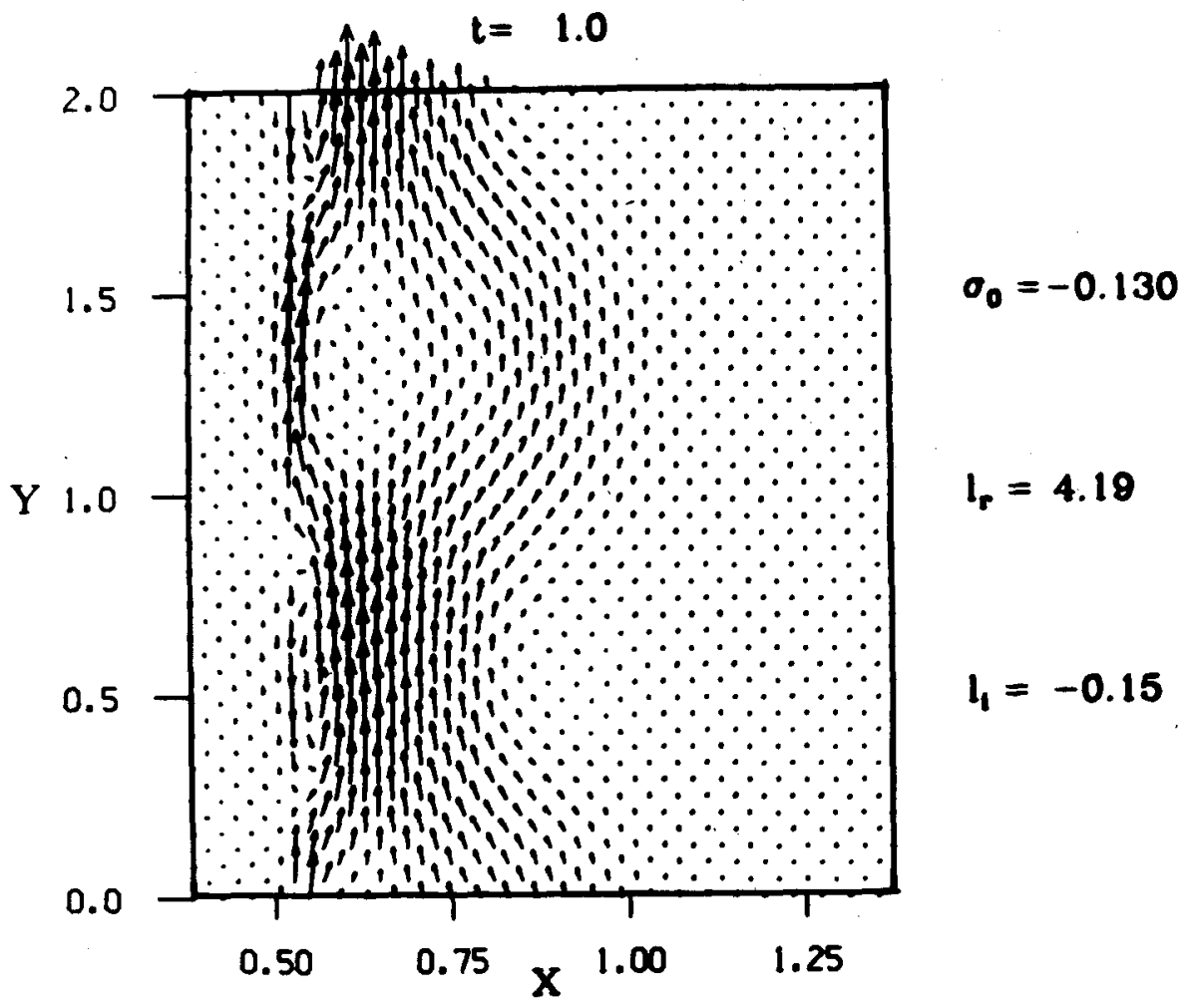

b

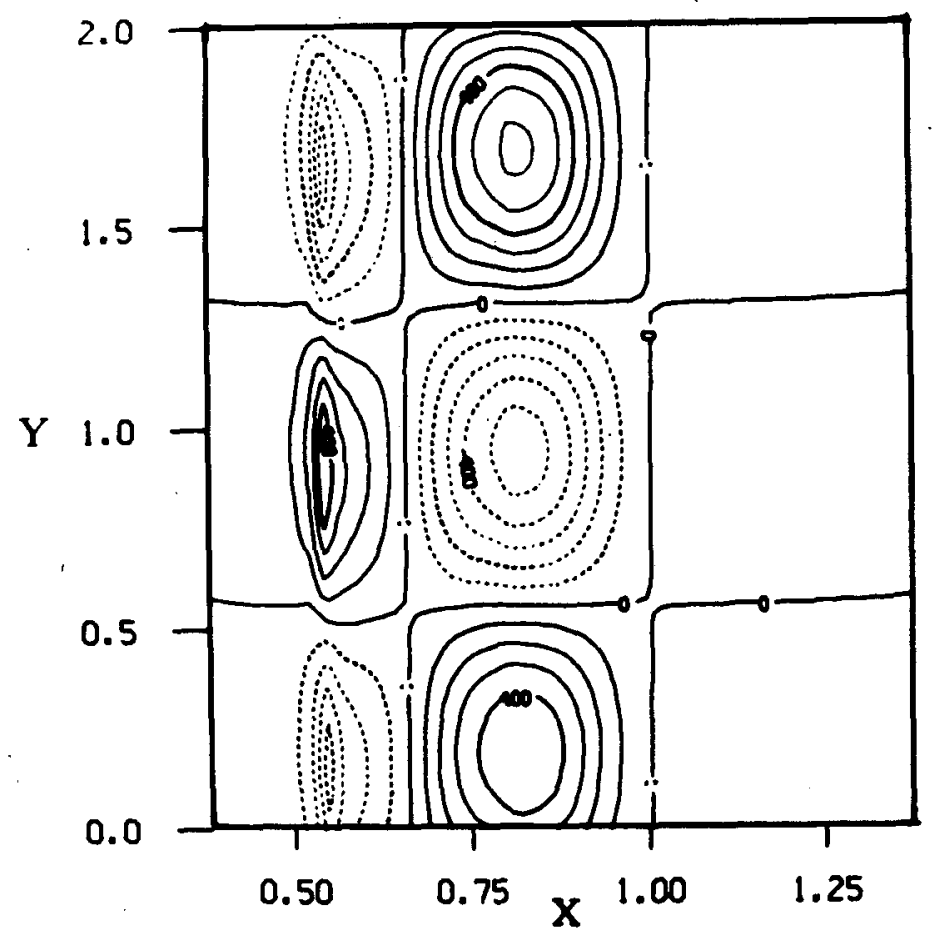

Fig. 13. As in Fig. 12 except $\zeta=-0.15$.

$$
\begin{aligned}
& \sigma_{0}=-0.130 \\
& I_{r}=4.19 \\
& l_{1}=-0.15
\end{aligned}
$$




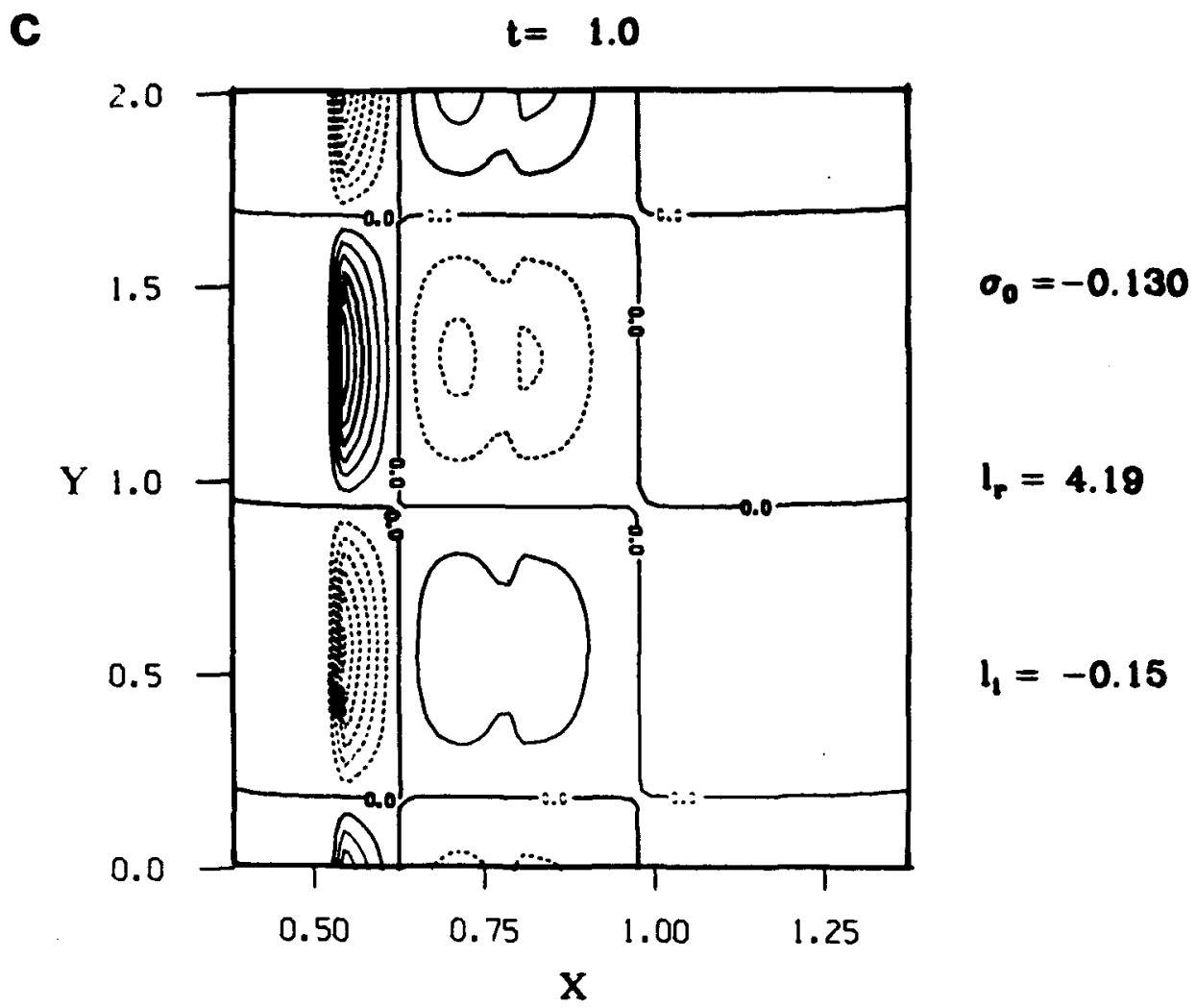

FiG. 13. (Continued)

propagation of a cold-dome meander by Chew et al. (1985). The deflection of the background current by the perturbation is greatest at the surface where $u$ has its maximum amplitude, and is coherent with depth (Fig. 13). This is consistent with observations by Bane et al. (1981) from an air-dropped XBT survey of the Gulf Stream, in which they found that the maximum deflection of the Stream occurred at the surface, and was coherent over the depth range of the AXBT probes. Bane et al. (1981) and Brooks and Bane (1981) also found that the maximum in cyclonic relative vorticity occurred in the trailing portion of a meander crest near where a warm filament joined the crest. The mode 2 wave reproduces this feature. The velocity and buoyancy fields in the small anticyclonic eddy that trails upstream of the meander crest in Fig. 12 imply the existence of a warm filament-like structure there that is separated from the main portion of the current by the cool dome of upwelled water. The anticyclonic circulation in this filament resembles that postulated by Pietrafesa and Janowitz (1980) as an interpretation of their current meter data. Data presented recently by Lee and Atkinson (1983) from the Gulf Stream between the Florida Straits and Cape Hatteras confirm the existence of a cyclonic circulation shoreward of a meander trough and an anticyclonic circulation seaward of a crest.
It certainly appears that the mode 2 wave reproduces many of the observed features of the 8-day wave, within the limits of linear theory. It is surprising that a linear theory indicates the presence of warm filaments trailing behind a meander crest. The formation of the filaments in the model is due to the presence of nodes in the cross-stream and vertical velocities and requires no lateral advection or entrainment of shelf water. The cooler water between the filament and the Stream is due to upwelling of water from below. Of course, this must be viewed only as the incipient stages of meander development, for as the perturbation amplitude grows, the nonlinear terms become important. It appears, however, that the features predicted by the linear theory do exist in finite amplitude in the real world.

It is not readily apparent why the mode 2 wave should be selected preferentially over the other modes. It is possible that the mode 2 wave has the highest growth rate, although this model was not able to isolate the maximum growth rates for any of the modes for reasons described above. The highest growth rates found here for the mode 2 wave give $e$-folding scales of about one wavelength. Eady (1949) found that the most unstable wave had a phase speed equal to the average speed of the background current, which is the case for the phase speed of the mode 2 wave 
corresponding to the observed 8-day wave; however, Eady's results were for a much simpler background configuration. The mode 2 wave does appear to have a larger bandwidth in the response spectra than the other modes. This implies that it may be more readily excited by a broad-band forcing such as an impulse. A small meander upstream of the Charleston Bump impinging on the Bump might be considered as such an impulse forcing; however, the triggering mechanism for the meanders is beyond the scope of this study.

\section{Conclusions}

Spatially unstable normal modes of oscillation are found in a realistic model of the Gulf Stream over the continental slope. The mode 2 wave closely resembles the observed 8-day wave, exhibiting close agreement in phase speed and in current and temperature structures. The description of the meander process that emerges from the model is consistent with that postulated by Webster (1961a) and accounts for many of the features observed in more recent studies, such as the formation of warm filaments and cold domes.

The instability mechanism for all modes is found to be of the mixed barotropic-baroclinic type. Baroclinic energy conversion dominates for all modes, with the ratio of potential energy conversion to kinetic energy conversion generally increasing with increasing mode number. All the normal modes found in this study are primarily surface trapped. The wave guide which supports these wave motions is the potential vorticity gradient induced by the sloping density surfaces, and is strongest near the surface. The effect of differing bottom topographic slopes or background fields on the normal modes has yet to be determined, but it is suspected that the normal mode structures are sensitive to the particular form of background configuration and topography chosen. A systematic variation of the background and topographic parameters is now necessary to investigate this dependence of the normal modes.

While the results of this model are highly suggestive, especially in regard to the observed 8-day wave, they are by no means conclusive. This model can make no inferences about the actual triggering mechanism for the waves. The neglect of the nonlinear terms is a severe constraint on the model, although many of the features of the linear theory do appear to persist in finite amplitude in the real world. The logical next step is to construct a fully nonlinear, time dependent model of the Gulf Stream from the Florida Straits to Cape Hatteras, including the effects of the Charleston Bump.

Acknowledgments. We would like to thank Professor James J. O'Brien and Professor Ya Hsueh for providing work space and computing facilities at the Florida State University for Dr. Luther during the completion of this research and for their many helpful discussions. We also thank the anonymous reviewers whose thoughtful comments made this a better manuscript. We extend our gratitude to Dr. John Adams of the National Center for Atmospheric Research for his help with the numerical method used here. This work was supported through the University of North Carolina Marine Sciences Program by the National Science Foundation under Grant OCE-79-23413 and by the Office of Naval Research under Contract N00014. C-0354. Additional support was provided through the Mesoscale Air-Sea Interaction Group at Florida State University by the Office of Naval Research under Contract N00014-80-C-0076. Computing services were provided by the National Center for Atmospheric Research, which is supported by the National Science Foundation.

\section{REFERENCES}

Adams, J., 1979: New craylib file: CROSEL. NCAR Computing Facility Notes, No. 60, 5.

Bane, J. M. Jr., and D. A. Brooks, 1979: Gulf Stream meanders along the continental margin from the Florida Straits to Cape Hatteras. Geophys. Res. Lett., 6, 280-282.

- and K. R. Lorenson, 1981: Synoptic observations of the three-dimensional structure and propagation of Gulf Stream meanders along the continental margin. J. Geophys. Res., 86, 6411-6425.

Bretherton, F. P., 1966a: Critical layer instability in baroclinic flows. Quart. J. Roy. Meteor. Soc., 92, 325-334.

- 1966b: Baroclinic instability and the short wave cut-off in terms of potential vorticity. Quart. J. Roy. Meteor. Soc., 92, 335-345.

Brooks, D. A., and J. M. Bane, Jr., 1978: Gulf Stream deflection by a bottom feature off Charleston, South Carolina. Science, 201, $1225-1226$

- and - 1981: Gulf Stream fluctuations and meanders over the Onslow Bay upper continental slope. J. Phys. Oceanogr., $11,247-256$.

Chao, S. Y., and G. S. Janowitz, 1979: The effect of a localized topographic irregularity on the flow of a boundary current along the continental margin. J. Phys. Oceanogr., 9, 900-910.

Charney, J. G., 1947: The dynamics of long waves in a baroclinic westerly current. J. Meteor., 4, 135-163.

Chew, F., J. M. Bane, Jr. and D. A. Brooks, 1985: On vertical motion, divergence, and the thermal wind balance in colddome meanders, a diagnostic study. J. Geophys. Res., (in press).

de Szoeke, R. A., 1975: Some effects of bottom topography on baroclinic instability. J. Mar. Res., 33, 93-122.

Eady, E. T., 1949: Long waves and cyclone waves. Tellus, 1, 3352.

Gaster, M., 1962: A note on a relation between temporally increasing and spatially increasing disturbances in hydrodynamic instability. J. Fluid Mech., 14, 222-224.

1965: On the generation of spatially growing waves in a boundary layer. J. Fluid Mech., 22, 433-441.

Gent, P. R., 1975: Baroclinic instability of a slowiy varying zonal flow. Part 2. J. Atmos. Sci, 32, 2094-2102.

Hart, J. E., 1974: On the mixed stability problem for quasigeostrophic ocean currents. J. Phys. Oceanogr., 4, 349-356.

Hogg, N. G., 1976: On spatially growing baroclinic waves in the ocean. J. Fluid Mech., 78, 217-235.

Holland, W. R., and D. B. Haidvogel, 1980: A parameter study of the mixed instability of idealized ocean currents. Dyn. Atmos. Oceans, 4, 185-215. 
Killworth, P. D., 1980: Barotropic and baroclinic instability in rotating stratified fluids. Dyn. Atmos. Oceans, 4, 143-184.

Lee, T. N., and L. P. Atkinson, 1983: Low-frequency current and temperature variability from Gulf Stream frontal eddies and atmospheric forcing along the U.S. outer continental shelf. $J$. Geophys. Res., 88, 4541-4568.

- , - and R. V. Legeckis, 1981: Observations of a Gulf Stream frontal eddy on the Georgia continental shelf, April, 1977. Deep Sea Res., 28, 347-378.

Legeckis, R. V., 1976: The influence of bottom topography on the path of the Gulf Stream at latitude $31 \mathrm{~N}$ from NOAA's satellite imagery. Eos Trans. $A G U, 27,260$.

- 1 1979: Satellite observations of the influence of bottom topography on the seaward deflection of the Gulf Stream off Charleston, South Carolina. J. Phys. Oceanogr., 9, 483-497.

McIntyre, M. E., 1970: On the non-separable baroclinic parallel flow instability problem. J. Fluid Mech., 40, 273-306.

Mooers, C. N. K., 1975: Several effects of a baroclinic current on the cross-stream propagation of inertial-internal waves. Geophys. Fluid Dyn., 6, 245-275.

Mysak, L. A., E. R. Johnson and W. W. Hsieh, 1981: Baroclinic and barotropic instabilities of coastal currents. J. Phys. Oceanogr., 11, 209-230.

Orlanski, I., 1969: The influence of bottom topography on the stability of jets in a baroclinic fluid. J. Atmos. Sci., 26, 12161232.

- and M. D. Cox, 1973: Baroclinic instability in ocean currents. Geophys. Fluid Dyn., 4, 297-332.

Pashinski, D. J., and G. A. Maul, 1973: Use of ocean temperature while coasting between the Straits of Florida and Cape Hatteras. Mar. Wea. Log, 17, 1-3.

Pedlosky, J., 1964a: The stability of currents in the atmosphere and the ocean. Part I. J. Atmos. Sci., 21, 201-219.

,$- 1964 \mathrm{~b}$ : The stability of currents in the atmosphere and the ocean. Part II. J. Atmos. Sci., 21, 342-353.

- , 1979: Geophysical Fluid Dynamics, Springer-Verlag, 617 pp. Pietrafesa, L. J., and G. S. Janowitz, 1981: On the dynamics of the Gulf Stream Front in the Carolina Capes. Proc. Second Int. Symp. on Stratified Flows, Trondhaim, Norway, Tapin Publishing Company, 184-197.

Richardson, W. S., W. J. Schmitz and P. P. Niiler, 1969: The velocity structure of the Florida Current from the Straits of Florida to Cape Fear. Deep Sea Res., 16, 225-231.

Salby, M. L., 1981: Rossby normal modes in nonuniform background configurations. Part I: Simple fields. Part II: Equinox and Solstice conditions. J. Atmos. Sci., 38, 1803-1840.

Schoeberl, M. R., and J. H. E. Clark, 1980: Resonant planetary waves in a spherical atmosphere. J. Atmos. Sci., 37, 20-28.

Watson, J.,1962: On spatially growing finite disturbances in plane Poiseuille flow. J. Fluid Mech., 14, 211-221.

Webster, F., 1961a: A description of Gulf Stream meanders off Onslow Bay. Deep Sea Res., 9, 130-143.

$-196 \mathrm{lb}$ : The effect of meanders on the kinetic energy balance of the Gulf Stream. Tellus, 13, 392-401.

Wright, D. G., 1980: On the stability of a fluid with a specialized density stratification. Part II: Mixed baroclinic-barotropic instability with application to the northeast Pacific. $J$. Phys. Oceanogr., 10, 1307-1322. 